\title{
Real-Time Hazard Detection and Avoidance Demonstration for a Planetary Lander
}

\author{
Dr. Chirold D. Epp ${ }^{1}$, Edward A. Robertson ${ }^{2}$, John M. Carson III ${ }^{3}$ \\ NASA, Johnson Space Center, Houston,Texas, 77058
}

\begin{abstract}
The Autonomous Landing Hazard Avoidance Technology (ALHAT) Project is chartered to develop and mature to a Technology Readiness Level (TRL) of six an autonomous system combining guidance, navigation and control with terrain sensing and recognition functions for crewed, cargo, and robotic planetary landing vehicles. In addition to precision landing close to a pre-mission defined landing location, the ALHAT System must be capable of autonomously identifying and avoiding surface hazards in real-time to enable a safe landing under any lighting conditions. This paper provides an overview of the recent results of the ALHAT closed loop hazard detection and avoidance flight demonstrations on the Morpheus Vertical Testbed (VTB) at the Kennedy Space Center, including results and lessons learned. This effort is also described in the context of a technology path in support of future crewed and robotic planetary exploration missions based upon the core sensing functions of the ALHAT system: Terrain Relative Navigation (TRN), Hazard Detection and Avoidance (HDA), and Hazard Relative Navigation (HRN).
\end{abstract}

\section{Nomenclature}

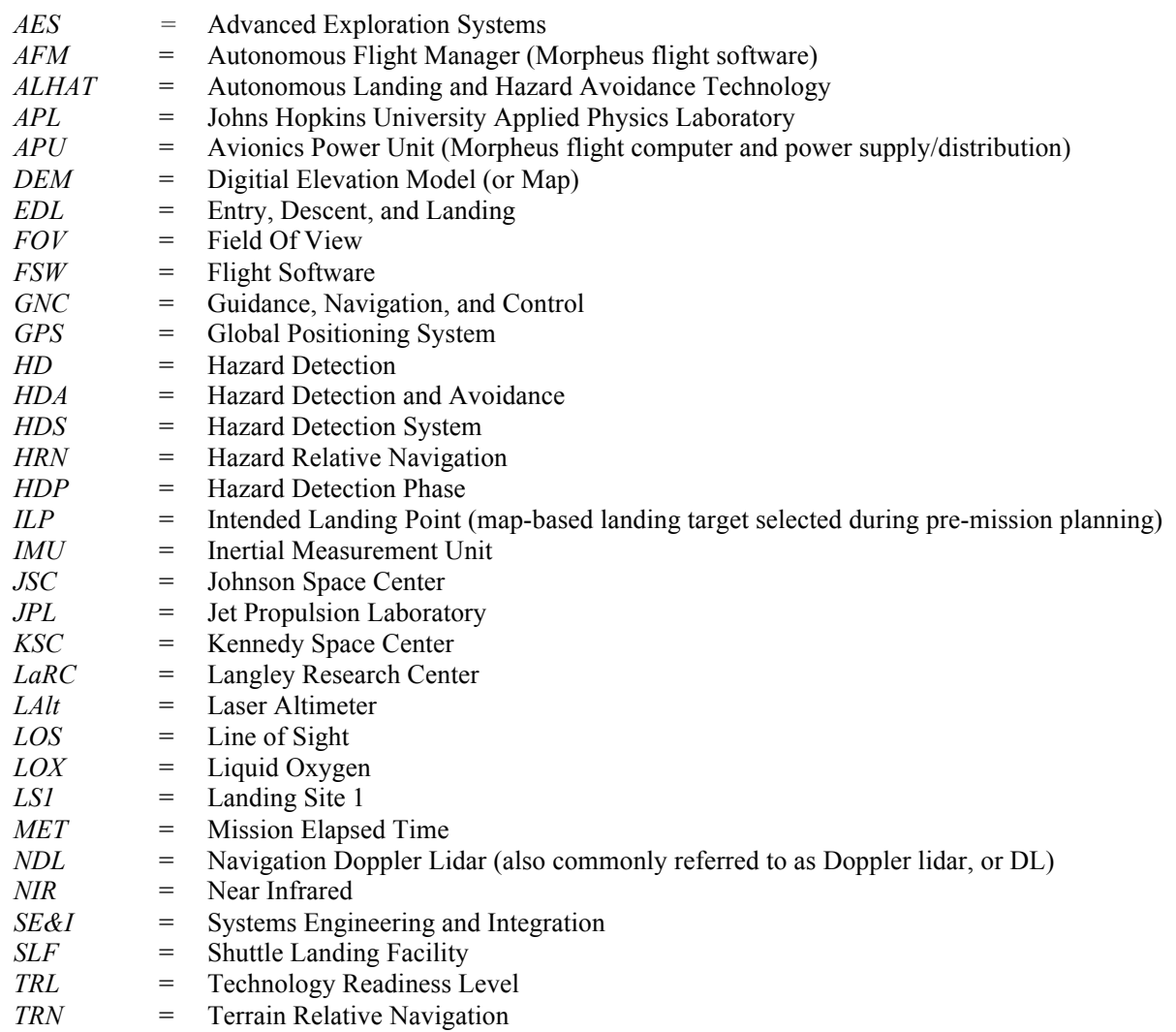

1 ALHAT Project Manager, Aerospace and Flight Mechanics Division, 2101 NASA Parkway, Houston, TX 77058/EG1, AIAA Senior Member.

2 Deputy ALHAT Project Manager, Aerospace and Flight Mechanics Division, 2101 NASA Parkway, Houston, TX 77058/EG5.

3 ALHAT SE\&I Lead, IPA Detail to JSC from JPL, Detailed to Aerospace and Flight Mechanics Division, 2101 NASA Parkway, Houston, TX 77058/EG1, AIAA Associate Fellow.

American Institute of Aeronautics and Astronautics 


\section{ALHAT Overview}

Autonomous precision landing enhanced by real-time hazard detection and avoidance has emerged as a technology of considerable interest in the U.S. and international aerospace communities over the past decade. Since the Autonomous Landing and Hazard Avoidance Technology Project (commonly known as ALHAT ${ }^{1,2}$ ) was chartered by NASA in 2006, the ALHAT team has focused on developing and maturing to a Technology Readiness Level of six a highly capable autonomous system combining guidance, navigation, and control (GNC) with terrain sensing and recognition functions for crewed, cargo, and robotic planetary landing vehicles. The ALHAT team evolved an entry, descent, and landing (EDL) approach that combines three techniques: Terrain Relative Navigation (TRN), Hazard Detection and Avoidance (HDA), and Hazard Relative Navigation (HRN). This general EDL strategy is designed to support a broad range of mission requirements and planetary landing destinations and can be tailored to meet the scope and scale of a particular mission.

The TRN function compares terrain data collected by onboard sensors with stored orbital reconnaissance maps to support accurate global navigation. TRN has long been employed in terrestrial military applications and, in 2009, the ALHAT Project successfully demonstrated several TRN techniques tailored for planetary spaceflight applications. ${ }^{3}$ TRN supports the ALHAT global landing accuracy requirement, which serves two primary functions. First, it facilitates scientific and engineering mission objectives involving precision landings near areas of interest defined by natural resources or other observable factors. Second, from a safe landing perspective, TRN positions a landing vehicle in close proximity to an area deemed favorable for finding one or more safe landing sites based upon premission analysis of available reconnaissance data. Although TRN is not strictly required for safe landing, the technique can significantly increase the probability of actively locating a suitable landing site, particularly within rugged planetary terrain. Even in the case of a "blind" landing, the probability of mission success is enhanced by the reduced landing dispersions provided by TRN. In addition, TRN can be accomplished with very modest lander resources. For all of these reasons, the ALHAT team views TRN as a critical navigation technique for future autonomous landers.

The HDA and HRN functions are the most challenging aspects of an autonomous safe landing system. The configuration chosen by ALHAT to demonstrate hazard detection (HD) uses an onboard linear mode flash lidar sensor mounted in a two-axis gimbal to generate a 3-D local terrain map during the descent trajectory. Depending on sensor performance, map generation can begin a kilometer or more from the map-based landing target selected by mission planners (referred to by ALHAT as the Intended Landing Point, or ILP). The Digital Elevation Model/Map (DEM) resulting from the mosaic of 3-D frames is then parsed to identify and rank the available safe landing locations based on the design characteristics and operational constraints of the lander. But identifying safe landing sites is only the first part of the HDA challenge. In order to avoid hazards and land near the selected safe site, it is critical to maintain a highly accurate, onboard, surface-relative navigation state in terms of position, velocity, and attitude. ALHAT utilizes the HRN function to supplement the ground-relative navigation data provided by high precision, laser-based altimeter and velocimeter sensors. The HRN function correlates flash lidar frames acquired during the latter stages of the approach phase with the DEM developed for hazard detection to provide position updates accurate to a fraction of a meter. Using an IMU to propagate this surface-relative navigation state knowledge, the vehicle can then descend to the planetary surface with minimal error over the final tens of seconds of the trajectory in the absence of terrain-relative sensor inputs. Dead-reckoning on an IMU during terminal descent eliminates the complications of attempting to acquire accurate sensor measurements through the dust field created by the descent engine plume.

The ALHAT team initially developed seven Level 0 requirements to drive the development of the ALHAT system. As shown in Table 1, the landing precision requirement was subsequently divided into global and local aspects to address the complementary, but distinct functions of navigating to a map-based planetary target (R0.003a) and landing in close proximity to a safe landing site identified in real-time during the descent trajectory (R0.003b). After thorough research and analysis, the ALHAT team determined that advancements in active, laser-based sensors capable of providing highly accurate ground-relative measurements of position, velocity, and 3-D topography were essential for satisfying three key components of the ALHAT Level 0 requirements: real-time HDA, local landing precision, and independence from ambient surface lighting conditions. In addition, a Hazard Detection System (HDS) that can rapidly map and process a large area of terrain for human exploration missions requires a highspeed, high-volume computing architecture significantly more capable than existing space-qualified processors. The ALHAT team chose to pursue the challenge of satisfying the Level 0 requirements for a human mission with the understanding that the results would be scalable and tailorable to less demanding exploration scenarios.

2

American Institute of Aeronautics and Astronautics 
Table 1. ALHAT Level 0 Requirements

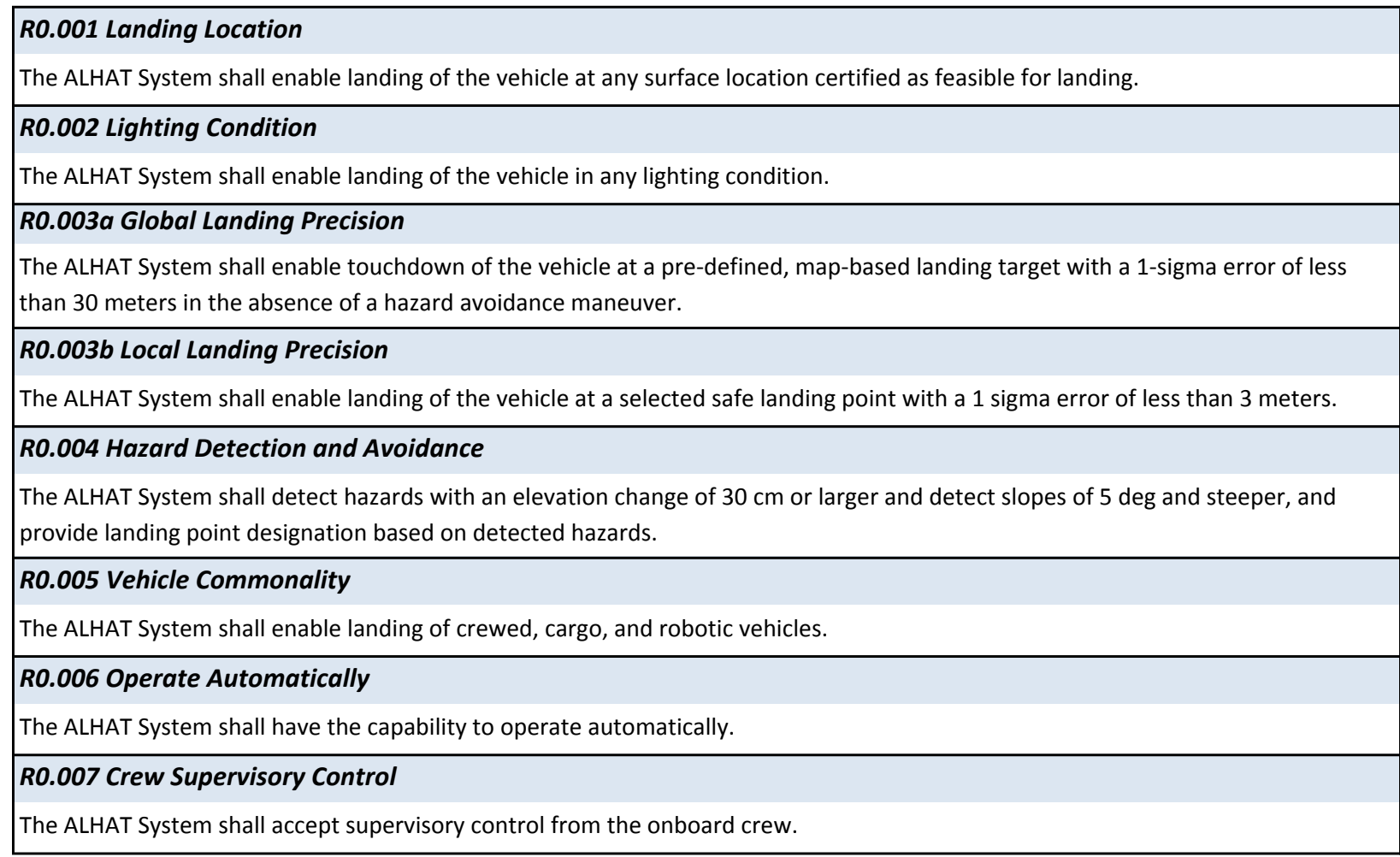

The ALHAT team pursued several parallel paths of research and development to satisfy the Level 0 requirements. The Jet Propulsion Laboratory (JPL) team focused primarily on the development, modeling, and simulation of TRN, HDA, and HRN algorithms and associated computing hardware. JPL also led the planning and execution of the ALHAT field tests from FY2006 through FY2009. The Johns Hopkins University Applied Physics Laboratory (APL) team developed a separate passive optical TRN approach along with new software for using scientific lunar rock and crater distribution models to synthetically enhance detail in reconnaissance maps to support high fidelity TRN and HDA simulations. APL also provided detailed scientific analyses of lunar terrain characteristics and ambient lighting conditions, as well as the capabilities and limitations of human vision for HDA. The Langley Research Center (LaRC) team focused on the development and testing of a new generation of lidar sensors to support TRN, HDA, and HRN, and also managed the contracts resulting from the NASA Research Announcement (NRA) solicitation released by the ALHAT project in FY2007. LaRC personnel were also critical in the rapid planning, integration and execution of the integrated ALHAT helicopter test in FY2012. ALHAT project management was located at the Johnson Space Center (JSC) along with the systems engineering and integration (SE\&I) and GNC development and simulation functions. The JSC team also served as the liaison between the Morpheus and ALHAT teams for the integration and testing of the ALHAT system with the Morpheus lander at JSC and KSC.

As summarized in Table 2, the ALHAT team led or partnered in the execution of twelve major field tests. These tests initially focused on sensor characterization, then evolved to address integrated sensor/software tests of the core TRN, HDA, HRN, and surface-relative navigation functions and, finally, culminated in autonomous demonstrations of real-time hazard detection and avoidance and precision landing on the Morpheus rocket-powered lander. Through analysis, simulation, test, and demonstration, the ALHAT team addressed all eight Level 0 requirements to varying degrees of fidelity, with a strong emphasis placed on integrated tests of sensors and software in relevant, dynamic flight environments. By focusing on eight simple and clearly defined requirements, the multicenter ALHAT team achieved substantial advances in EDL technology development and demonstration over a period of eight years that spanned the retirement of the Shuttle program and the cancellation of the Constellation program, as well as significant shifts in human exploration objectives and budget priorities. 
Table 2. Summary of ALHAT Field Test Activities

\begin{tabular}{|c|c|c|c|c|c|}
\hline Test ID & Date & $\begin{array}{c}\text { Test } \\
\text { Objective }\end{array}$ & Sensors & $\begin{array}{c}\text { Test } \\
\text { Platform }\end{array}$ & Description \\
\hline FTO & $\begin{array}{c}\text { September } \\
2006\end{array}$ & HDA & Scanning Lidar & Helicopter & Characterization of a scanning lidar sensor \\
\hline JIGSAW & $\begin{array}{c}\text { September } \\
2007\end{array}$ & HDA & MIT Lincoln Lab & Helicopter & $\begin{array}{l}\text { JIGSAW helicopter field test of MIT Lincoln Lab flash lidar } \\
\text { (test not managed by ALHAT, data classified after test) }\end{array}$ \\
\hline FT1 & $\begin{array}{l}\text { May } \\
2008\end{array}$ & HDA/HRN & $\begin{array}{c}\text { Flash Lidar } \\
\text { Optical Camera }\end{array}$ & Helicopter & $\begin{array}{l}\text { Early characterization of COTS flash lidar sensor and HDA/HRN } \\
\text { algorithms; TRN using COTS passive optical camera }\end{array}$ \\
\hline FT2 & $\begin{array}{c}\text { August } \\
2008\end{array}$ & $\begin{array}{l}\text { Navigation } \\
\text { Sensor }\end{array}$ & Doppler Lidar & Helicopter & $\begin{array}{l}\text { Early characterization of the ALHAT Doppler lidar sensor and } \\
\text { algorithm for highly accurate ground-relative navigation }\end{array}$ \\
\hline FT3 & $\begin{array}{l}\text { March } \\
2009\end{array}$ & TRN & $\begin{array}{c}\text { Flash, Doppler, } \\
\text { Altimeter, Cameras }\end{array}$ & $\begin{array}{l}\text { Fixed-wing } \\
\text { Aircraft }\end{array}$ & $\begin{array}{l}\text { TRN test of ALHAT flash/altimeter lidar sensors \& COTS passive } \\
\text { optical cameras; Doppler lidar sensor test }\end{array}$ \\
\hline FT4 & $\begin{array}{l}\text { August } \\
2009\end{array}$ & HDA/HRN & $\begin{array}{l}\text { Flash, Doppler, } \\
\text { Altimeter }\end{array}$ & Helicopter & $\begin{array}{l}\text { Integrated HDA/HRN system test using ALHAT flash lidar with } \\
\text { real-time, onboard data processing; Doppler lidar and altimeter }\end{array}$ \\
\hline FT5 & $\begin{array}{l}\text { December } \\
2012\end{array}$ & HDA/HRN & ALHAT System & Helicopter & $\begin{array}{l}\text { Real-time, quasi-closed loop field test of second generation } \\
\text { prototype HDA/HRN system developed for Morpheus flights }\end{array}$ \\
\hline Free Flight 10 & April 2, 2014 & $\begin{array}{l}\text { Open Loop } \\
\text { Evaluation }\end{array}$ & ALHAT System & $\begin{array}{l}\text { Morpheus } \\
\text { VTB }\end{array}$ & $\begin{array}{l}\text { Morpheus flight of second generation prototype ALHAT system } \\
\text { Morpheus navigation used for flight control and HDS pointing }\end{array}$ \\
\hline Free Flight 11 & April 24, 2014 & $\begin{array}{l}\text { Open Loop } \\
\text { Refinement }\end{array}$ & ALHAT System & $\begin{array}{l}\text { Morpheus } \\
\text { VTB }\end{array}$ & $\begin{array}{l}\text { Morpheus flight of second generation prototype ALHAT system } \\
\text { Morpheus navigation used for flight control and HDS pointing }\end{array}$ \\
\hline Free Flight 12 & April 30, 2014 & $\begin{array}{l}\text { Open Loop } \\
\text { Refinement }\end{array}$ & ALHAT System & $\begin{array}{l}\text { Morpheus } \\
\text { VTB }\end{array}$ & $\begin{array}{l}\text { Morpheus flight of second generation prototype ALHAT system } \\
\text { Morpheus navigation used for flight control and HDS pointing }\end{array}$ \\
\hline Free Flight 13 & May 22, 2014 & $\begin{array}{c}\text { Closed Loop } \\
\text { Demonstration }\end{array}$ & ALHAT System & $\begin{array}{l}\text { Morpheus } \\
\text { VTB }\end{array}$ & $\begin{array}{l}\text { Morpheus flight of second generation prototype ALHAT system } \\
\text { ALHAT navigation used for flight control and HDS pointing }\end{array}$ \\
\hline Free Flight 14 & May 29, 2014 & $\begin{array}{c}\text { Closed Loop } \\
\text { Demonstration }\end{array}$ & ALHAT System & $\begin{array}{l}\text { Morpheus } \\
\text { VTB }\end{array}$ & $\begin{array}{l}\text { Morpheus flight of second generation prototype ALHAT system } \\
\text { ALHAT navigation used for flight control and HDS pointing }\end{array}$ \\
\hline
\end{tabular}

In the fourth quarter of FY2011, NASA Headquarters directed the Morpheus and ALHAT projects to join forces to demonstrate autonomous, real-time HDA and precision landing on the Morpheus rocket-powered lander. During the remainder of FY2011 and FY2012, the two teams integrated the ALHAT system with the Morpheus vehicle, completed a series of tether tests, and developed a large, high-fidelity hazard field of lunar analog terrain to support Morpheus/ALHAT safe landing demonstrations at the NASA Kennedy Space Center (KSC). ${ }^{3}$ As shown in Figure 1, KSC saved a considerable amount of money by constructing the highly durable, 100-meter by 100-meter hazard field using "crawler fines" for fill material and ground cover, as well as to build up the craters and slope features. Twenty-four craters were constructed ranging from $2.0 \mathrm{~m}$ to $4.8 \mathrm{~m}$ in diameter with depths of $0.20 \mathrm{~m}$ to $0.96 \mathrm{~m}$. Concrete debris from demolition projects was salvaged to form several hundred rock features varying in height from $30 \mathrm{~cm}$ to $100 \mathrm{~cm}$ with a typical height-to-width ratio of $1: 2 .{ }^{4}$ Crawler fines - crushed gravel created by the massive treads of the crawlers used to transport the Saturn V and the Space Shuttle vehicles - form a hard crust that inhibits plant growth and is highly resistant to wind and water erosion. Both of the 10-meter diameter concrete landing pads were covered with a thin layer of crawler fines for the Morpheus/ALHAT flights to present the flash lidar with terrain of reasonably uniform reflectivity. As visible in Figure 1 (top), the exhaust plume from the Morpheus main engine generates a dust cloud during descent starting at around $30 \mathrm{~m}$ altitude.

Although the Morpheus 1.5a vehicle was lost during its second free flight in August 2012, the Morpheus and ALHAT teams were prepared to execute a rapid turnaround. Due to the risks associated with the early Morpheus 1.5a free flights, mass simulators were employed in place of the actual ALHAT sensors, avionics, and power systems. In addition, the Morpheus team had already begun development of a second lander vehicle, Morpheus $1.5 \mathrm{~b}$, based on the same general design, but incorporating a number of small improvements based upon operational experience with the original vehicle. As a result, the combined team was able to assemble Morpheus $1.5 b$, fully integrate the ALHAT system, and begin tether testing at the NASA Johnson Space Center (JSC) in roughly eighteen months. 

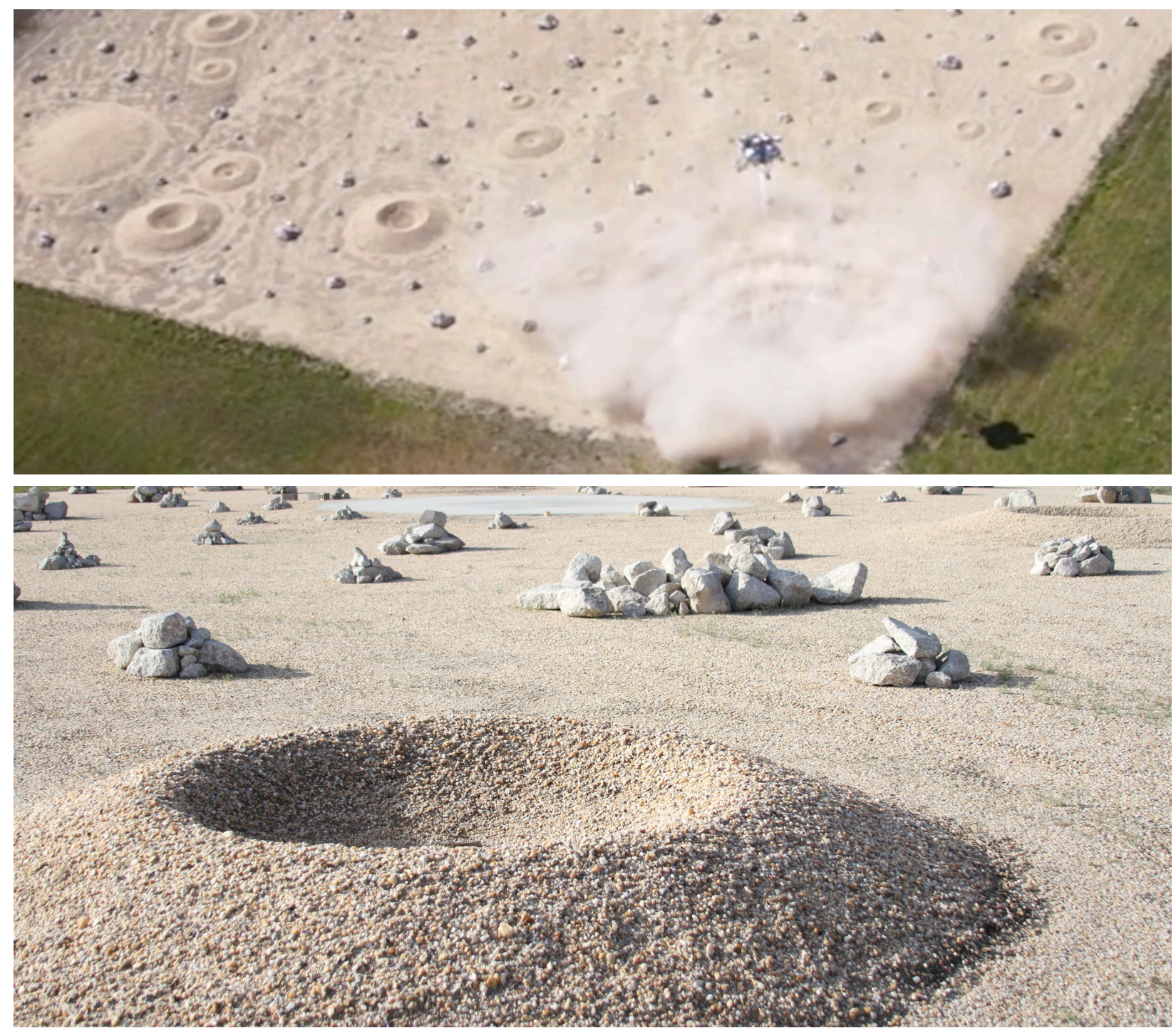

Figure 1. Lunar Analog Terrain Constructed at the North End of the KSC SLF

The Morpheus lander was designed for a lunar mission in terms of tank sizing and engine thrust. As a result, its performance as a terrestrial testbed is constrained by Earth's stronger gravitational field, even after engine upgrades were incorporated to increase its maximum thrust to nearly $22 \mathrm{kN}(5000 \mathrm{lbf})$. As usable propellant was displaced by growth in dry mass, the size and duration of the Hazard Detection Phase (HDP) trajectory envelope supported by Morpheus gradually decreased from the original goal of a $1000 \mathrm{~m}$ initial slant range and a flight duration of 120 seconds or greater. The team eventually compromised on the HDP trajectory displayed in Figures 2 and 3, with a groundtrack distance of $424 \mathrm{~m}$ to the Initial Landing Point 1 (ILP1) and $405 \mathrm{~m}$ to the center of the near landing pad, designated Landing Site 1 (LS1). ILP1 is the pre-defined (and unsafe) landing target for Morpheus and also represents the approximate center of the DEM generated by the HDS. The nominal HDP trajectory reaches a maximum altitude of approximately $245 \mathrm{~m}$, provides an initial slant range at the start of HDS terrain mapping of roughly $460 \mathrm{~m}$, and has a total flight duration of approximately 98 seconds. The team allocated a total of 14.1 seconds to the HDS to generate the DEM, identify and rank the top five safe landing sites, and return the safe site list to the Morpheus Autonomous Flight Manager (AFM) software for validation against truth data. At the end of that 14.1 second interval, roughly $150 \mathrm{~m}$ downtrack from the launch pad in the nominal HDP trajectory, the Morpheus lander begins a blend maneuver to divert towards LS1. If the safe site selected by the ALHAT HDS is valid (within $2 \mathrm{~m}$ radius of LS1), then the AFM accepts the HDS site as the new landing target. If the HDS does not 
provide a site within the allocated interval or the identified top ranked HDS site is not valid, then the AFM uses the surveyed truth location of LS1 as the new landing target.

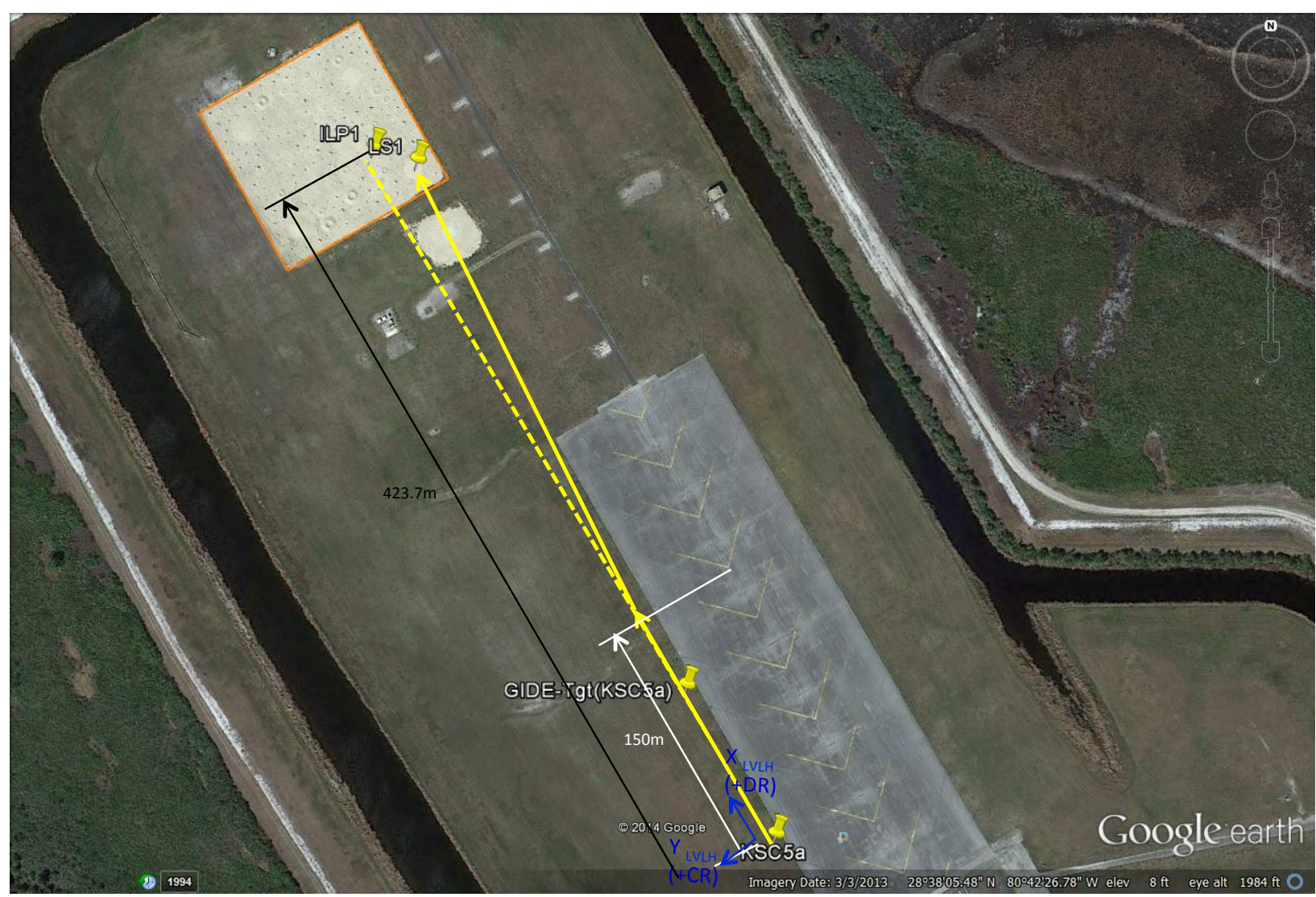

Figure 2. Configuration of the SLF Test Area for the Morpheus/ALHAT Flights

Figure 3 shows the expansion of the Morpheus $1.5 \mathrm{~b}$ free flight trajectory profiles (altitude vs. crossrange in meters) from FF3 through FF14. Prior to FF10, Morpheus 1.5b carried mass simulators in place of the ALHAT sensors, avionics, and power system. For FF10 through FF14, the five longest and highest trajectories shown tightly overlapped in Figure 3, the ALHAT system was installed and fully operational on Morpheus 1.5b.

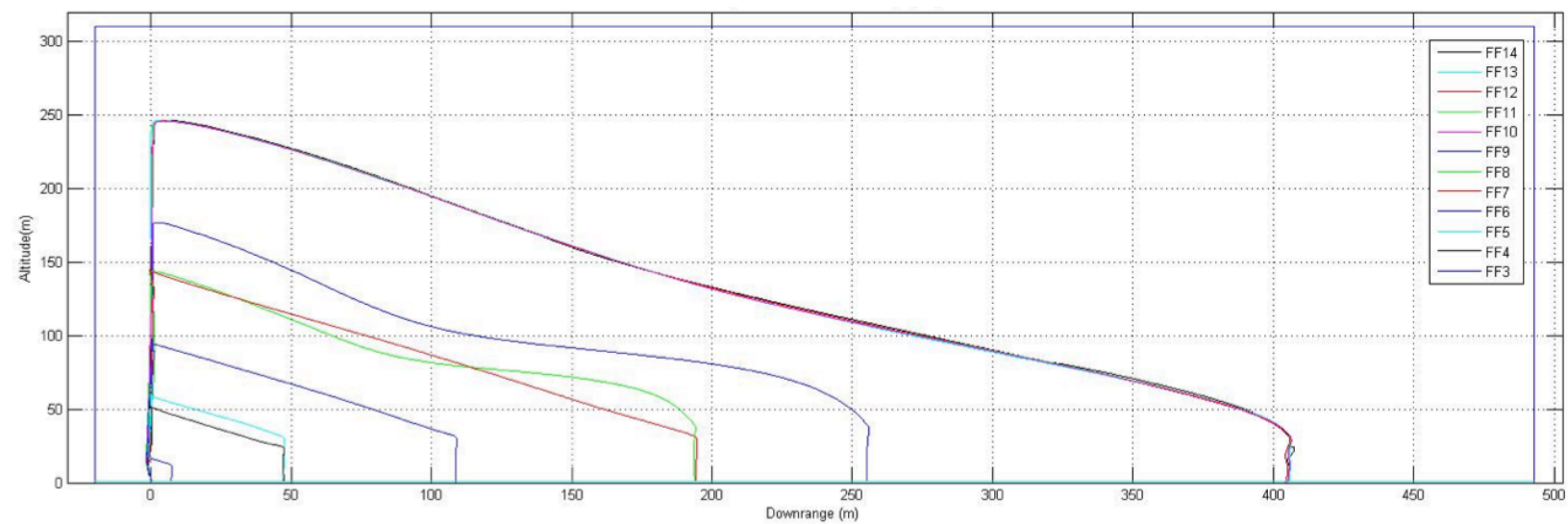

Figure 3. Free Flight Trajectory Expansion 


\section{ALHAT INTEGRATION WITH THE MORPHEUS LANDER}

A block diagram illustrating the electrical interfaces between the Morpheus and ALHAT systems is provided in Figure 4. The color coding of the components highlight the multi-center organization of the Morpheus and ALHAT projects. The only top-level Morpheus/ALHAT interface change since the ALHAT integration with Morpheus 1.5a is the addition of voltage monitoring circuits between the HDS Battery Box and the Morpheus Avionics and Power Unit (APU). Adding these three signals to the APU telemetry stream provided the control room with insight into the battery charge state for the HDS gimbal, flash lidar, and compute element circuits.

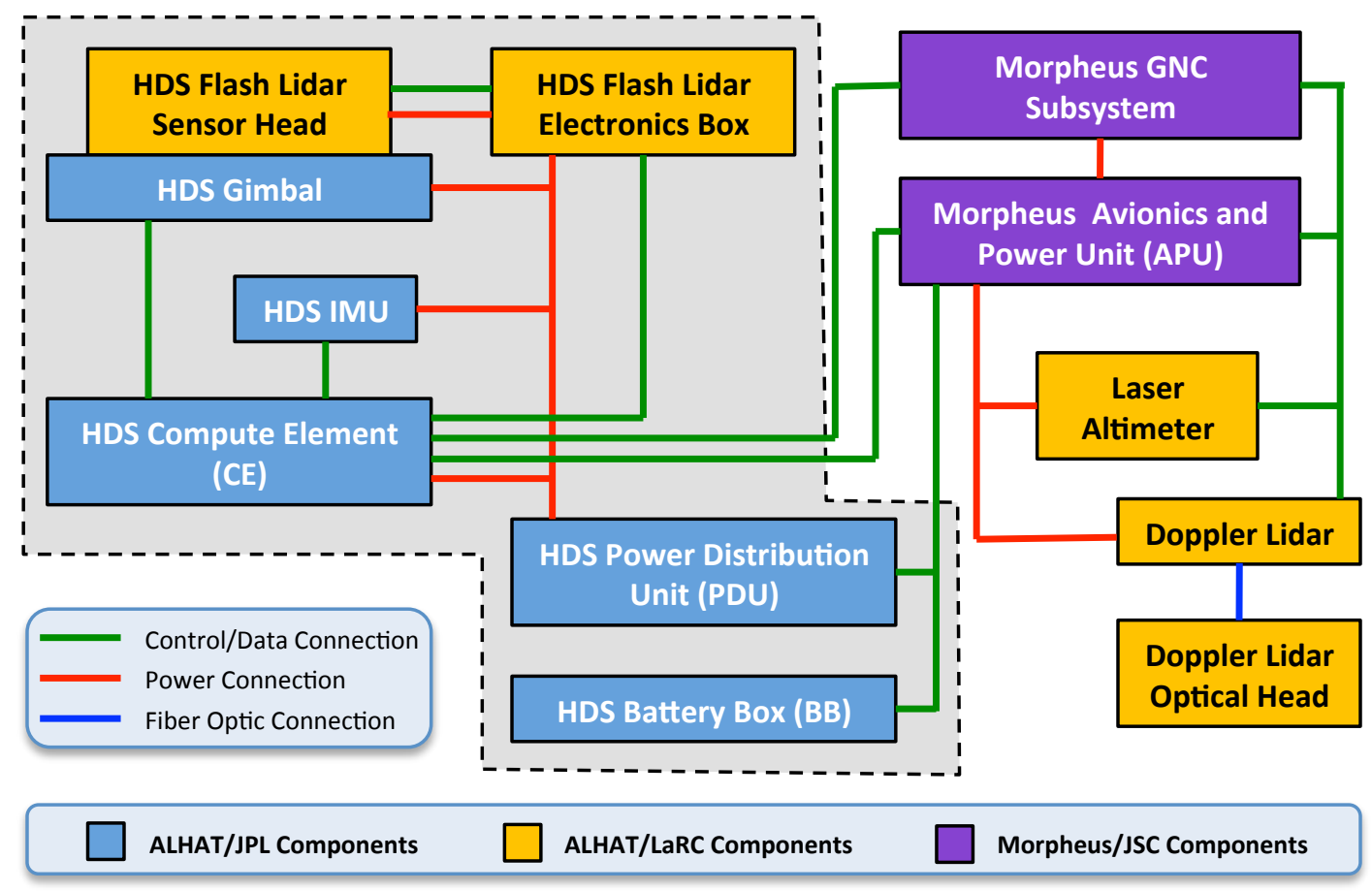

Figure 4. ALHAT/Morpheus Interface Block Diagram

The HDS consists of the components located within the gray box in Figure 4. The command and control interfaces between the HDS and the Morpheus vehicle include the vehicle navigation state, pulse-per-second (PPS) timing signal, AFM moding commands, HDS safe site information, and a range of health and status telemetry. The HDS Compute Element (CE) interfaces with the flash lidar sensor, directs the gimbal, contructs the DEM, develops the hazard probability map, and identifies/ranks the safe sites. Only the HDS end product - the ranked safe site list - is sent to the Morpheus AFM for action. To simplify the interfaces between two complex systems that were developed in parallel, the HDS includes its own electrical power and distribution system. For spaceflight applications, the HDS and host vehicle will likely use an integrated power system to reduce size and mass.

The Morpheus Avionics and Power Unit (APU) executes two extended Kalman navigation filters in parallel. The Morpheus navigation filter processes inputs from GPS, IMU, and a short-range COTS laser altimeter. The ALHAT navigation filter uses the Morpheus IMU data, but substitutes the ALHAT laser altimeter for the Morpheus COTS unit. The ALHAT navigation filter also processes ALHAT Doppler lidar velocimetry measurements and HRN position updates. During pre-flight operations, the Morpheus navigation solution is passed to the ALHAT navigation filter as a crossfeed measurement. The crossfeed is turned off prior to liftoff

From an integration standpoint, the ALHAT laser altimeter and Doppler lidar navigation sensors were treated as Morpheus elements. All of the power and data interfaces for these two sensors are routed to the Morpheus APU and GNC subsystem in support of the parallel navigation filter approach.

The Morpheus body coordinate system designates the vehicle centerline as the $\mathrm{X}$-axis with $+\mathrm{X}$ upwards. The liquid methane tanks are located along the $\mathrm{Y}$-axis with $+\mathrm{Y}$ to the right. The liquid oxygen tanks are located along the $\mathrm{Z}$ axis with $+Z$ in the forward direction of travel to complete the right-hand coordinate system. For reference, the $+Z$ 
oxygen tank is visible in the foreground of Figure 5(a) with the HDS gimbal assembly cantilevered from the Morpheus upper deck in the $+\mathrm{Y} /+\mathrm{Z}$ quadrant. The forward and outboard location of the HDS gimbal assembly was selected to provide a broad field of regard for the flash lidar sensor, both laterally and vertically, to facilitate terrain imaging during HDA and HRN. In practice, however, the Morpheus lander proved to be quite stable in attitude during the approach phase, requiring only modest articulation of the HDS gimbal to complete the mosaic while also compensating for vehicle motion.

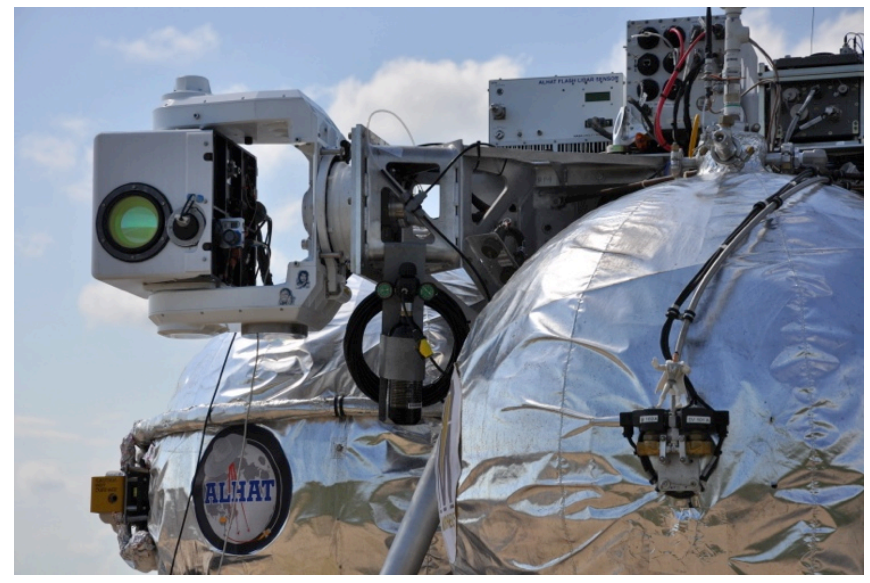

(a) Forward View

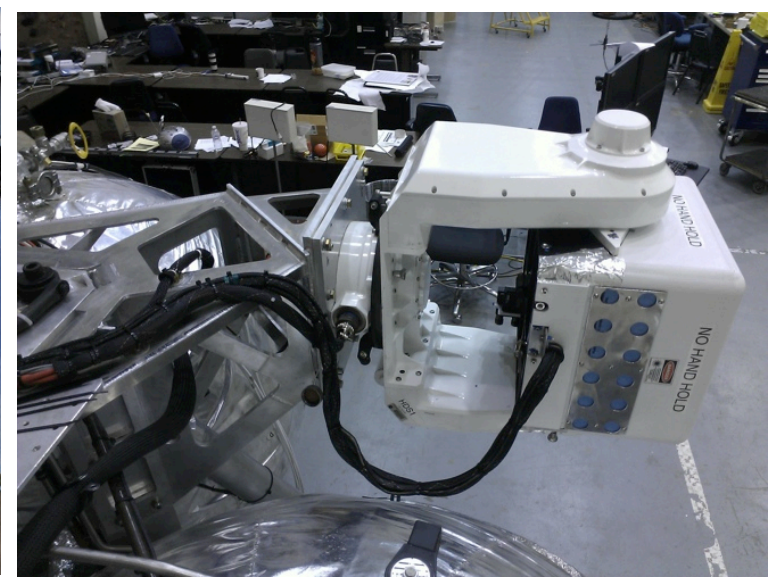

(b) Aft View

Figure 5. Gimbaled Flash Lidar Sensor Mounted on Morpheus 1.5b

In FY2008, the ALHAT project was directed to support the Constellation program in the implementation of safe landing system technologies to support human lunar exploration missions. The Constellation requirement for global lunar access meant that the ALHAT system must be able to locate suitable safe landing sites in rugged terrain with poor lighting conditions, such as near the lunar poles. In combination with the rugged terrain, the large diameter of the Altair lander meant that the ALHAT system would have to be capable of rapidly imaging and analyzing a large area of lunar terrain in order to maintain a high probability of finding a safe site. As a result, the ALHAT team began analyzing DEM sizes of 90x90m and 180x180m and investigating beam steering technologies applicable to the generation of large mosaics. For the purposes of landing system technology development, a two-axis gimbal appeared to be the most expedient and lowest risk approach for demonstrating the core HDS functions. However, it should be noted that there there are other approaches for lidar beam steering that may offer mass, size, and power advantages for spaceflight missions. For some robotic missions, beam steering may not even be required.

In a gimbaled system, power and data signals are generally routed to a connector at the base of the gimbal and then through slip rings to the instrument. However, there were concerns about the power quality that could be delivered to the Fibertek laser through the gimbal slip rings. In addition, the slip rings could only support a subset of the desired signals. As a result, a separate, external wire harness was routed to the flash lidar sensor head via a service loop, as shown in Figure 5(b). This service loop is quite large because it was sized to support the full pan/tilt range of the gimbal to its mechanical hardstops. This protected the wire harness from damage in the event of a violation of the more restrictive software-imposed pan/tilt limits. The service loop did not appear to adversely impact the performance of the HDS during flight.

The linear mode flash lidar currently employed by ALHAT is a modified version of the TigerEye sensor developed by Applied Scientific Concepts, Inc. LaRC installed a Class IV Fiberset laser $(1064 \mathrm{~nm}, 50 \mathrm{~mJ})$ to quadruple its operational range and also carefully calibrated the sensor to improve its range measurement noise, frame-to-frame range consistency, and range/intensity performance. As shown in Figure 5(a), the TigerEye employs separate transmit/receive optics - the receive lens is uncovered in this photo while the smaller transmit lens just to its right is capped for eye-safety during preflight operations. In FY2009, the ALHAT team explored the use of collinear transmit/receive optics and zoom lenses for flash lidar sensors. Collinear optics avoid parallax issues and are advantageous for compact beam steering techniques, such as mirrors and prisms. Zoom or stepped optics can be used to extend the operational range of a flash lidar for TRN, perform multiple cycles of HD at increasing 
resolutions, and/or expand the sensor field of view at lower altitudes for HRN. Both collinear optics and zoom/stepped lenses may be of benefit for future spaceflight missions that employ flash lidar sensors.

The ALHAT HDS uses an LN200 IMU to enable highly accurate gimbal pointing during the HDA mosaic. This small sensor is barely visible on the back side of the gimbal mount in Figure 5(a). If the host vehicle can provide navigation state updates to the HDS at a sufficiently high rate, this additional IMU is not required.

The remaining HDS components are located on the Morpheus 1.5b upper deck. From left to right in Figure 6(a), the HDS Flash Lidar Electronics Box, HDS Compute Element and the Morpheus Space Integrated GPS INS (SIGI) are visible near the front of the upper deck. The tall aluminum frame in the background to the far right supports the Doppler Lidar Chassis. In Figure 6(b), the HDS Battery Box is stacked on top of the HDS Power Distribution Unit (PDU) near the center of the photo. The box to the far left is the Morpheus Avionics and Power Unit (APU).

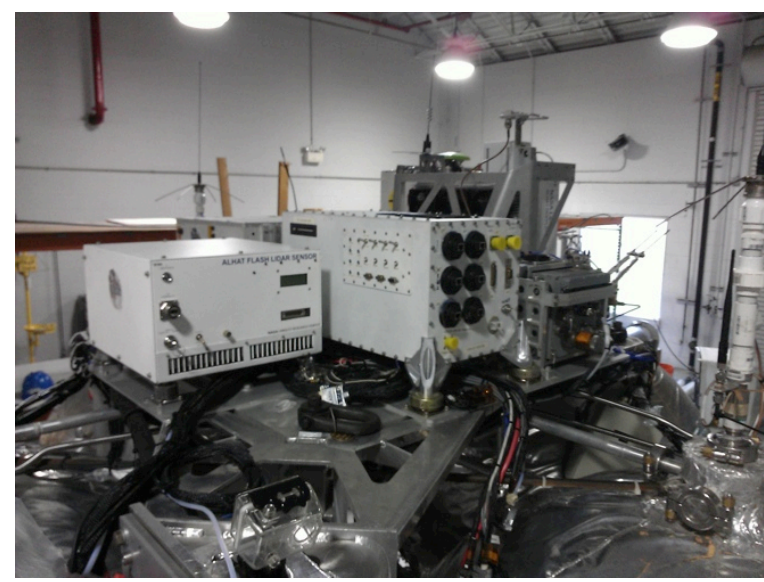

(a) Forward View

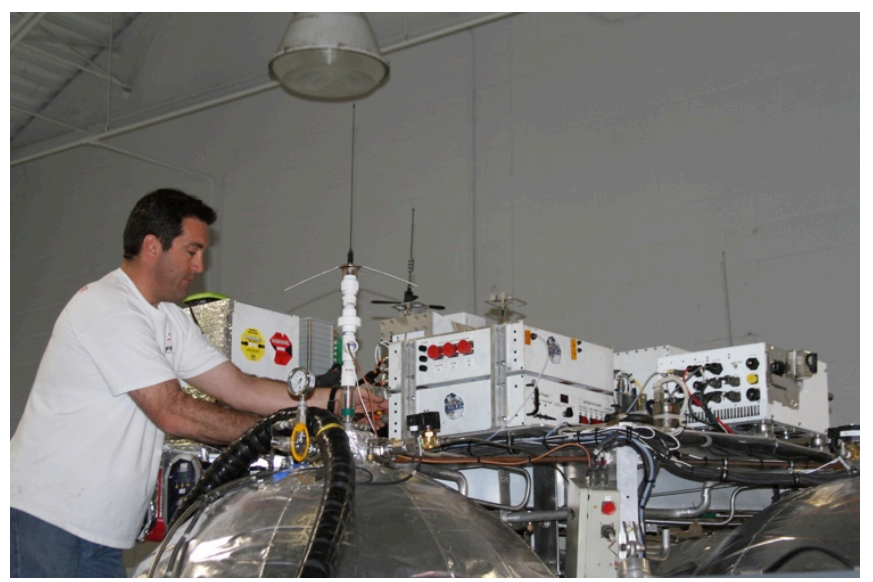

(b) Aft View

Figure 6. Components on the Morpheus Upper Deck

The ALHAT team wanted to mount the ALHAT Doppler lidar sensor head and laser altimeter sensor on or near the upper deck to provide separation of the sensors from the Morpheus main engine and associated vibration, acoustics, and thermal environments at the base of the lander. However, these sensors require an unobstructed view of the ground and the Morpheus cruciform tank and leg configuration greatly restricts nadir lines of sight from the upper deck. The three-beam Doppler lidar optical head posed a particular challenge because the three beams are canted $22.5^{\circ}$ from nadir and spaced symmetrically at $120^{\circ}$ intervals about a central shaft. For the FY2014 free flight campaigns, these two sensors were mounted in separate locations outboard of the lower deck surrounded by propellant tanks and the landing gear struts. In Figure 7, the Doppler lidar optical head is visible below the Morpheus APU in the $-\mathrm{Y} /-\mathrm{Z}$ quadrant of the vehicle. This location was chosen to help trim the vehicle center of gravity. The cylindrical baffles protruding beneath the Doppler sensor head were added to help protect the optics from debris impacts and the base thermal environment. The performance of the Doppler lidar sensor, in particular, was adversely impacted by its mounting location near the Morpheus main engine. The laser altimeter is visible to the right in the $+\mathrm{Y} /-\mathrm{Z}$ quadrant of the vehicle, partially hidden by the main strut of a leg.

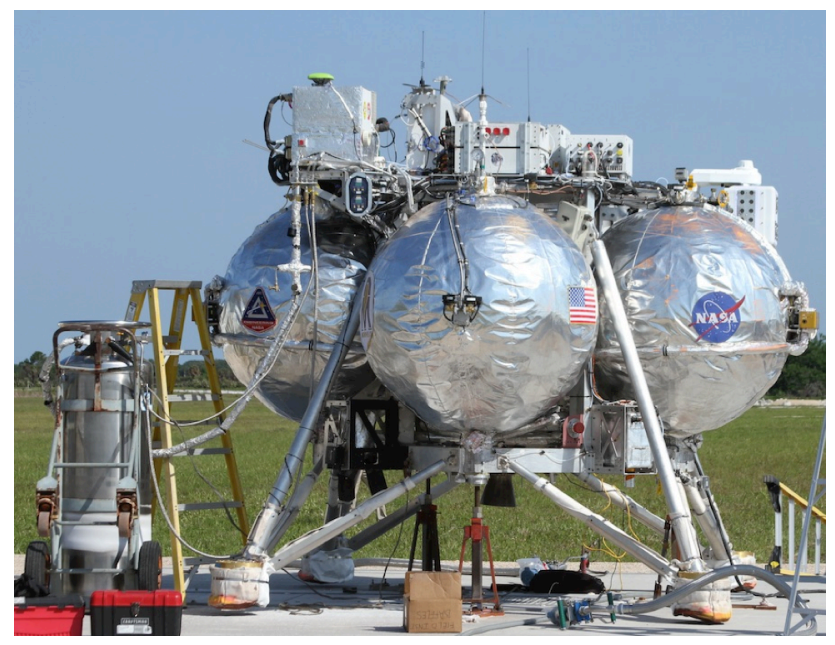

Figure 7 Doppler Lidar and Laser Altimeter Sensors 


\section{ALHAT FLIGHT OBJECTIVES AND PLANNING}

The fundamental objective for the integrated Morpheus/ALHAT free flight campaigns was to successfully demonstrate real-time, autonomous HDA followed by a controlled, accurate landing near the selected safe site during a representative, closed loop, terrestrial free flight on a rocket-powered lander. Successful demonstration of these capabilities using the ALHAT navigation filter would serve to elevate the tested ALHAT safe landing system and its associated functions, components, and software to TRL 6. In order to achieve success at the integrated level, each ALHAT component must also perform at a satisfactory level in cooperation with the rest of the ALHAT system and the Morpheus host vehicle. The detailed test objectives for the HDS functions, ALHAT navigation filter, and individual ALHAT lidar sensors are described below.

\section{Hazard Detection System (HDS)}

- HDS pointing with respect to the GIDE target satisfies the accuracy specification

- HDS pointing with respect to the Lidar target satisfies the accuracy specification

- HDS provides accurate pointing to the selected HRN feature

- Gimbal performance satisfies static and dynamic pointing requirements

- HDS meets safe site identification and ranking objectives using Morpheus navigation

- HDS meets safe site identification and ranking objectives using ALHAT navigation

- DEM is successfully generated within six seconds of HDA initiation

- DEM map-tie error is less than one meter

- HDS generates an accurate DEM that is highly representative of the hazard field truth data

- Safe sites are identified appropriately relative to the actual hazard field

- Safe site rankings show appropriate discrimination and distribution

- Top ranked safe site is located on the landing pad

- Top ranked safe site is with a two meter radius of the center of the landing pad (threshold imposed by Morpheus)

Hazard Relative Navigation (HRN)

- Strong DEM reference feature selected for HRN correlations

- HRN reference feature is maintained within the flash lidar FOV during HRN operations

- Valid and accurate HRN measurements are produced during the relevant operational region of the approach trajectory

- HRN measurements improve the ALHAT navigation state estimate

- HRN produces valid measurements on VTB nav

- HRN produces valid measurements on ALHAT navigation

Flash Lidar (FL)

- FL maintains full operational status after multiple exposures to the Morpheus flight environment

- Automatic Gain Correction (AGC) maintains images within the dynamic range of the flash lidar sensor

- FL maintains its internal subsystems within their defined operational environmental bounds

- FL operation is independent of ambient lighting conditions

- FL sensitivity is sufficient to produce high-quality range images at the maximum slant range provided during the Morpheus HDP free flight trajectory

- FL images demonstrate the low levels of range measurement noise required for successful detection of small hazards and the identification and ranking of safe sites

- FL range accuracy is adequate for HRN measurements

- FL range and intensity images are free from pixel pre-triggering 
Navigation Doppler Lidar (NDL)

- NDL maintains full operational status after multiple exposures to the Morpheus flight environment

- NDL performance is demonstrated to be independent of ambient lighting conditions

- NDL provides a high degree of measurement data availability throughout the approach trajectory

- NDL provides superior data quality with the precision of beam LOS velocities within $3 \mathrm{~cm} / \mathrm{s}, 1$-sigma

\section{Laser Altimeter (LAlt)}

- LAlt maintains full operational status after multiple exposures to the Morpheus flight environment

- LAlt performance is demonstrated to be independent of lighting conditions

- LAlt provides a high degree of measurement data availability throughout the approach trajectory

- LAlt provides superior data quality with a LOS range measurement error within $25 \mathrm{~cm}, 1$-sigma

\section{ALHAT Navigation Filter}

- ALHAT Nav correctly processes LAlt measurements

- ALHAT Nav correctly processes NDL measurements

- ALHAT Nav correctly processes HRN measurements

- Error for the ALHAT Nav estimated position relative to Morpheus Nav remains within the established error tolerance threshold during the entire HDP trajectory

- Error for the ALHAT Nav estimated velocity relative to Morpheus Nav remains within the established error tolerance threshold during the entire HDP trajectory

- Error growth in the ALHAT Nav estimated attitude is sufficiently limited to support the execution of the HDA and HRN and achieve a safe, controlled landing

The GNC moding approach evolved during the free flight campaigns as the team gained experience with the ALHAT system and progressed towards the objective of fully closing the loop on ALHAT navigation and satisfying the objectives listed above. Figure 10 summarizes the GNC and AFM moding configuration used during FF14 for the Prime Nav, VTB Crossfeed, ALHAT measurements, HDS UPP Source (used for HDS pointing), and AFM HDS Commands. For FF14, ALHAT nav was used to fly the vehicle except in the event of a soft or hard abort, and VTB crossfeed was turned off just prior to liftoff. The ALHAT LAlt measurements were only processed by ALHAT nav from the FlightAcent through the FlightApproachFinal modes. Similarly, the NDL velocity measurements are only processed during a subset of the approach trajectory. To avoid excess measurement noise from the concentration of dust and hot, turbulent air during liftoff and landing, the ALHAT nav does not process the LAlt and NDL measurement when the vehicle is in close proximity to the ground. This is consistent with the established ALHAT approach of using dead-reckoning during terminal descent. The NDL LOS range measurements (labeled DL_Alt in Figure 8) were not processed by ALHAT nav during FF14 because the processing of these measurements proved to be problematic during FF10 and FF11 and a fix has not been found. HRN processing is enabled during the appropriate flight modes following DEM generation and concluding as the vehicle reached final approach. The pointing source for the HDS was designated to be the ALHAT nav. However, it should be noted that in the current version of the AFM flight software, the HDS UPP Source automatically changes to VTB nav if the AFM commands a Prime Nav downmode. The Prime Nav downmode occurred very late in the FF14 trajectory and, thus, did not affect HDS operations. In FF13, however, the downmode to VTB nav occurred during Flight Approach and affected HDS pointing during HRN measurements. The AFM HDS Command simply modes the HDS from standby (noop or "no operations") through Prepare, Operate, and Site Index, back to noop, and then shutdown during terminal descent. The Operate command instructs the HDS to generate the DEM and develop the safe site list. The HDS then sends the Site Index to AFM for the validation of the top-ranked safe site as the new landing target. Following the conclusion of the HRN measurements, the HDS goes back into standby and then shuts down. The HDS shut down sequence tilts the flash lidar sensor head up $30^{\circ}$ above the horizontal to help protect its optics from landing debris. For future Morpheus/ALHAT free flights, the expectation is that the processing of NDL velocity measurements will begin earlier in ascent. In addition, the cutoff altitude for the LAlt and NDL measurements will be raised to $30 \mathrm{~m}$ rather than the current $22 \mathrm{~m}$ (FlightDescent1 in Figure 8) to eliminate noisy readings. It is currently unclear whether or not the NDL LOS range measurements will be processed by ALHAT nav in future free flights. 


\begin{tabular}{|c|c|c|c|c|c|c|c|c|}
\hline \multicolumn{6}{|c|}{ Nominal Closed-Loop Testing for FREEFLIGHT14 } & \multirow[b]{2}{*}{ HRN } & \multirow[b]{2}{*}{ HDS UPP Src } & \multirow[b]{2}{*}{ AFM HDS cmd } \\
\hline & Prime Nav & $\begin{array}{c}\text { VTB } \\
\text { Crossfeed }\end{array}$ & LA_ALT & DL_Alt & DL_Vel & & & \\
\hline Off & $\mathrm{n} / \mathrm{a}$ & $\mathrm{n} / \mathrm{a}$ & Off & Off & Off & Off & $\mathrm{n} / \mathrm{a}$ & noop \\
\hline NavStartup & $\mathrm{n} / \mathrm{a}$ & $\mathrm{n} / \mathrm{a}$ & Off & Off & Off & Off & $\mathrm{n} / \mathrm{a}$ & \\
\hline NavOn & ALHAT & On & Off & Off & Off & Off & ALHAT & noop \\
\hline GNC Actuator Tests & ALHAT & On & Off & Off & Off & Off & ALHAT & noop \\
\hline GuidOn & ALHAT & On & Off & Off & Off & Off & ALHAT & noop \\
\hline CntrIOnStandby & ALHAT & On & Off & Off & Off & Off & ALHAT & noop \\
\hline IgnSeq & ALHAT & On & Off & Off & Off & Off & ALHAT & noop \\
\hline FlightAscent & ALHAT & Off & On & Off & Off & Off & ALHAT & noop \\
\hline FlightHDSPrep & ALHAT & Off & On & Off & Off & Off & ALHAT & prepare \\
\hline FlightHDA & ALHAT & Off & On & Off & On & On & ALHAT & operate \\
\hline FlightApproach & ALHAT & Off & On & Off & On & On & ALHAT & site_index \\
\hline FlightApproachFinal $(166 \mathrm{~m})$ & ALHAT & Off & On & Off & On & Off & ALHAT & noop \\
\hline FlightDescent1 $(22 \mathrm{~m}$ alt) & ALHAT & Off & Off & Off & Off & Off & ALHAT & noop \\
\hline FlightDescent2 (15m alt) & ALHAT & Off & Off & Off & Off & Off & ALHAT & noop \\
\hline FlightDescentFinal $(4 \mathrm{~m})$ & ALHAT & Off & Off & Off & Off & Off & ALHAT & shutdown \\
\hline AbortSoft & VTB & Off & On & Off & On & Off & ALHAT & shutdown \\
\hline EngineShutdown & ALHAT & On & Off & Off & Off & Off & ALHAT & shutdown \\
\hline AbortHard & VTB & Off & Off & Off & Off & Off & ALHAT & shutdown \\
\hline
\end{tabular}

Figure 8. GNC and AFM Moding Configuration for FF14

Figure 8 is a plot of the GNC modes and HDS modes versus mission elapsed time (MET) for FF14. During FF14, the HDS operation extended from roughly 43 seconds to 69 seconds MET and the processing of ALHAT NDL and LAlt measurements continued until approximately 91.6 seconds MET. The number and sequence of Morpheus GNC modes and ALHAT HDS modes are not expected to change for future Morpheus/ALHAT free flights. However, variations in the trajectory, such as flying a longer distance to ILP2/landing pad 2, will likely impact the duration and MET of these modes. The ALHAT nav processing modes for the ALHAT navigation sensors are expected to change as the issues identified in FF10 through FF14 are addressed and solutions are implemented.

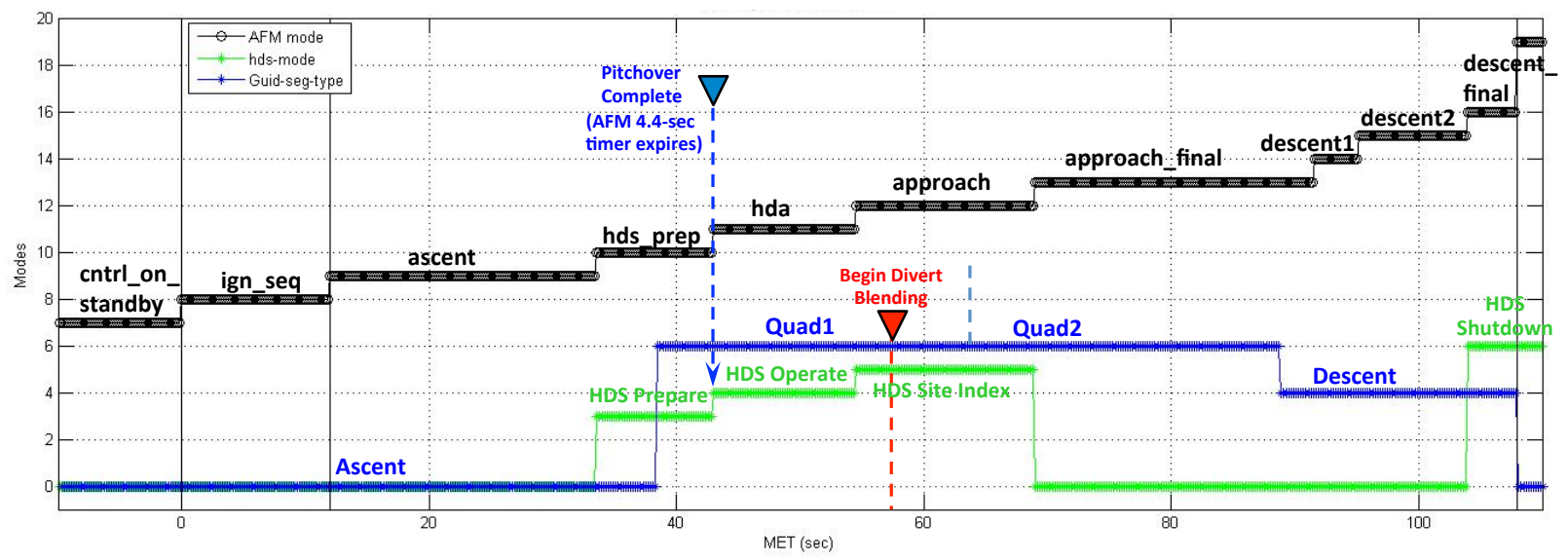

Figure 9. FF14 Modes Versus Mission Elapsed Time (MET) 


\section{OVERVIEW OF MORPHEUS/ALHAT FREE FLIGHTS}

The Morpheus/ALHAT GNC configurations for FF10 through FF14 are shown in Figure 10. The color coding in the table indicates changes between flights. FF10 marked the first time that the Morpheus/ALHAT system executed an HDP trajectory and collected ALHAT sensor data at relevant altitudes and velocities in the induced environment produced by the Morpheus vehicle. The first three Morpheus/ALHAT free flights, FF10-FF12, used the VTB navigation solution both to control the vehicle trajectory and as an input to the HDS to drive the pointing of the gimbaled flash lidar sensor. During these flights, the ALHAT system collected and processed HDS and navigation data in "open loop" mode to determine how well the Morpheus/ALHAT system would perform if guidance and control were closed on the ALHAT navigation solution. Each flight represented a learning experience for the team with respect to the performance of the sensors in the induced flight environment and the interactions between the Morpheus and ALHAT systems. The third free flight, FF12, marked a milestone when the Morpheus AFM accepted and validated the safe site input from the ALHAT HDS and landed at that location. The final two flights closed the loop on the ALHAT navigation solution for control of the vehicle trajectory and as the input to the HDS for DEM generation, safe site selection, and HRN.

The Morpheus APU processes two navigation filters in parallel - VTB nav and ALHAT nav. VTB nav, which employs GPS, IMU, and altimeter measurements, was tested and refined during dozens of tether tests and nine free flights prior to ALHAT integration at KSC. As a result, VTB nav is relied upon as a close approximation of the vehicle "truth" state. Comparisons between the Morpheus and ALHAT navigation solutions are used to monitor the performance of ALHAT during free flights, and VTB nav serves as the backup in the event of a problem. In order to keep the two navigation filters in close agreement, the team employed a "crossfeed" technique in which the VTB navigation solution is fed into the ALHAT navigation filter as a measurement. The AFM directed the ALHAT navigation filter to stop processing the crossfeed measurement at the AFM mode listed in the "VTB Crossfeed" row

\begin{tabular}{|c|c|c|c|c|c|c|}
\hline & FF10 & FF11 & FF12 & FF13 & FF14 & Next Flight \\
\hline \multicolumn{7}{|l|}{ AFM Nav/Sensor Moding } \\
\hline Vehicle Nav Source & $\begin{array}{c}\text { Morpheus VTB } \\
\text { (ALHAT Open Loop) }\end{array}$ & $\begin{array}{c}\text { Morpheus VTB } \\
\text { (ALHAT Open Loop) }\end{array}$ & $\begin{array}{c}\text { Morpheus VTB } \\
\text { (ALHAT Open Loop) }\end{array}$ & ALHAT Closed Loop & ALHAT Closed Loop & ALHAT Closed Loop \\
\hline VTB Crossfeed & $\begin{array}{c}\text { Crossfeed on until } \\
\text { after FlightHDA mode }\end{array}$ & $\begin{array}{c}\text { Crossfeed on until } \\
\text { after FlightHDSPrep } \\
\text { mode } \\
\end{array}$ & $\begin{array}{c}\text { Crossfeed on until } \\
\text { after GNClgnSeq } \\
\text { mode }\end{array}$ & $\begin{array}{c}\text { Crossfeed on until } \\
\text { after GNClgnSeq } \\
\text { mode }\end{array}$ & $\begin{array}{l}\text { Crossfeed on until } \\
\text { after GNClgnSeq } \\
\text { mode }\end{array}$ & \\
\hline Laser Altimeter & $\begin{array}{c}\text { Used Ascent through } \\
\text { Shutdown }\end{array}$ & $\begin{array}{c}\text { Used Ascent through } \\
\text { Shutdown }\end{array}$ & $\begin{array}{c}\text { Inhibitied at } \\
\text { DescentFinal }(3 \mathrm{~m})\end{array}$ & Inhibitied at $22 \mathrm{~m}$ & Inhibitied at $22 \mathrm{~m}$ & \\
\hline Doppler Lidar Velocity & $\begin{array}{l}\text { Used Ascent through } \\
\text { Shutdown }\end{array}$ & $\begin{array}{l}\text { Used Ascent through } \\
\text { Shutdown }\end{array}$ & $\begin{array}{c}\text { Post-Mosaic until } \\
\text { Inhibitied at Descent2 } \\
(30 \mathrm{~m})\end{array}$ & $\begin{array}{l}\text { Post-Mosaic until } \\
\text { Inhibitied at } 22 \mathrm{~m}\end{array}$ & $\begin{array}{c}\text { Post-Mosaic until } \\
\text { Inhibitied at } 22 \mathrm{~m} \\
\text { Only processed with } 3 \\
\text { good beams }\end{array}$ & \\
\hline Doppler Lidar Altimetry & $\begin{array}{c}\text { Used Ascent through } \\
\text { Shutdown }\end{array}$ & $\begin{array}{c}\text { Used Ascent through } \\
\text { Shutdown }\end{array}$ & No longer used & No longer used & No longer used & \\
\hline HDS Pointing Source & Pointed on VTB Nav & Pointed on VTB Nav & $\begin{array}{l}\text { Pointed on VTB Nav } \\
\text { (Intended to point on } \\
\text { ALHAT Nav) }\end{array}$ & $\begin{array}{l}\text { Pointed on ALHAT } \\
\text { Nav until switch } \\
\text { (Intended to point on } \\
\text { ALHAT Nav } \\
\text { throughout) } \\
\end{array}$ & $\begin{array}{l}\text { Pointed on ALHAT } \\
\text { Nav until switch } \\
\text { (Intended to point on } \\
\text { ALHAT Nav } \\
\text { throughout) }\end{array}$ & \\
\hline HDS Site Selection & $\begin{array}{l}\text { Inhibited } \\
\text { (3.35 m from LS) }\end{array}$ & $\begin{array}{l}\text { Inhibited } \\
\text { (0.77 m from LS) }\end{array}$ & $\begin{array}{l}\text { Enabled } \\
(1.40 \mathrm{~m} \text { from LS) }\end{array}$ & \begin{tabular}{|l} 
Enabled \\
$(0.35 \mathrm{~m}$ from $\mathrm{LS})$ \\
\end{tabular} & $\begin{array}{l}\text { Enabled } \\
(2.55 \mathrm{~m} \text { from } \mathrm{LS})\end{array}$ & Enabled \\
\hline HRN & Pointed on VTB Nav & Pointed on VTB Nav & $\begin{array}{l}\text { Pointed on VTB Nav } \\
\text { (Intended to point on } \\
\text { ALHAT Nav) }\end{array}$ & $\begin{array}{l}\text { Pointed on ALHAT } \\
\text { Nav until switch } \\
\text { (Intended to point on } \\
\text { ALHAT Nav } \\
\text { throughout) } \\
\end{array}$ & $\begin{array}{l}\text { Pointed on ALHAT } \\
\text { Nav until switch } \\
\text { (Intended to point on } \\
\text { ALHAT Nav } \\
\text { throughout) }\end{array}$ & \\
\hline $\begin{array}{l}\text { AFM Nav Difference } \\
\text { Corridor }\end{array}$ & $\mathrm{N} / \mathrm{A}$ & N/A & N/A & $\begin{array}{l}1.5 \% \text { slant range, } \\
2.5 \mathrm{~m} \text { limit } \\
\text { Inhibited below } 15 \mathrm{~m} \\
\text { altitude }\end{array}$ & $\begin{array}{l}1.7 \% \text { slant range } \\
+2.1 \text { bias, } \\
2.5 \mathrm{~m} \text { limit } \\
\text { Inhibited below } 15 \mathrm{~m} \\
\text { altitude }\end{array}$ & Same corridor \\
\hline
\end{tabular}

Figure 10. Summary of Morpheus and ALHAT GNC System Configurations for the KSC Free Flights 
of Figure 8. The crossfeed technique was felt to be the best way to initialize the ALHAT nav during launch and maintain close agreement with VTB nav during ascent. However, the crossfeed approach also resulted in timing/latency issues in state comparisons between the two filters. The VTB nav measurements effectively included a time lag of roughly $180 \mathrm{msec}$ compared to the incoming ALHAT navigation sensor measurements. During dynamic flight, this latency manifests as phase shifts in the velocity and position estimates. This is not a problem for the VTB nav since the GPS measurement dominates and the latency effect diminishes as the vehicle slows down and begins its terminal descent. But this latency had a significant adverse impact on the ALHAT navigation solution and its comparison with VTB nav. Based on the analysis of flight data from FF10 and FF11, the AFM mode trigger was revised so that ALHAT nav stopped processing the VTB crossfeed information just prior to launch.

Flying in a terrestrial environment proved to be a much bigger challenge than anticipated. The ALHAT lidar sensors are affected by dust kicked up by launch and landing, as well as by the mass of turbulent, hot air created by the Morpheus main engine exhaust. This dust attenuated the signals of the Doppler lidar and laser altimeter sensors during launch and landing, resulting in significant measurement noise and even signal loss. Depending on the winds of the day, the dust cloud created during launch can also drift between the vehicle and the hazard field during the descending approach trajectory, causing pretriggering of pixels in the flash lidar range image. This reduces the quality of the flash lidar images for hazard detection and, in some cases, renders the images unusable for generating HRN measurements. After the first two Morpheus/ALHAT free flights, the area around the launch pad was watered down just prior to liftoff to minimize the dust cloud. But even a reduced amount of dust can cause a problem if the winds blow the particles in front of the ALHAT sensors. So a wind placard was implemented for FF13 and FF14 to restrict the direction and velocity of the prevailing winds at liftoff. It should be noted that other airborne particulates, such as pollen or smoke from grass fires, can also cause pixel pretriggering in flash lidar sensor images. However, the dust, pollen, or smoke particles must be fairly close to the flash lidar sensor - a few tens of meters - in order to produce a sufficiently strong signal to trigger a detector pixel. A mitigation approach is to implement a time delay (commonly referred to as a range gate) before the flash lidar sensor begins processing return signals. The ALHAT team attempted to implement a range gate on the modified TigerEye sensor, but the feature had to be removed because it interfered with the calibration of the instrument. However, it is highly recommended that future generations of flash lidar sensors intended for EDL applications include a user-configurable range gate.

The other major environmental problem for the ALHAT lidar sensors was the adverse effect of the large mass of hot air generated by the exhaust plume of the Morpheus main engine. With unfavorable winds, this hot air could rise between the vehicle and the hazard field and degrade the output from the flash lidar. The ALHAT flash lidar operates in the near-infrared (NIR) part of the spectrum very close to visible light, and the heated turbulent air caused by the Morpheus engine can produce a blurring effect analogous to the heat shimmer visible over hot ground in the daytime. The hot air had a much more serious effect on the performance of the NDL sensor, which is located much closer to the Morpheus engine. Rather than pointing ahead of the vehicle at a shallow angle like the flash lidar, the three NDL beams point downwards and slightly away from nadir. Depending on the relative wind direction and velocity (a function of the prevailing wind combined with vehicle motion) at various points during a flight, the mas of hot air often resulted in a loss of signal from one or two beams, and occasionally all three beams. This had a major effect on the quantity of velocity data available for the ALHAT navigation filter. After several flights, it became clear that the velocity estimates obtained from the current implementation of the ALHAT navigation filter were excellent when all three NDL beams were available, but drifted away from the VTB nav estimate when only one or two NDL beams were providing data. Because of the tight position corridor imposed on the VTB versus ALHAT nav comparison, even a small velocity drift could quickly result in a violation of the boundary of the navigation comparison corridor. As a result, the navigation filter was revised to process NDL measurements for FF13 and FF14 only when all three beams were available. This occurred only for a short period of time during the latter portion of the approach phase and, unfortunately, this limited availability of the NDL was not quite sufficient to keep the ALHAT navigation solution within the corridor in the absence of good HRN measurements. Since each NDL beam provide very accurate data, it is unclear why the availability of only one or two beams should result in divergence of the ALHAT navigation velocity estimate. This is one of the key post-flight issues that is under assessment and must be fixed before another Morpheus/ALHAT flight campaign can occur. It is not known at this time if the problem is in the output of the NDL, the manner in which the NDL measurements are processed in the ALHAT navigation filter, or a combination of the two. For future Morpheus/ALHAT flights, the team plans to redesign the NDL optical head and relocate it to a higher and forward mounting location on the vehicle. This should greatly improve the availability of all three NDL beams during the approach trajectory. In addition, the same wind placard will benefit both the flash lidar and the NDL sensors. 
Although the Morpheus/ALHAT flights have focused on the velocimetry data provided by the NDL, each beam of this instrument is also capable of providing LOS range measurements. When LOS range measurements from all three NDL beams are available, the instrument can estimate the vehicle altitude and surface-relative attitude. The LOS range measurements from the NDL were processed by the ALHAT navigation filter during the first two flights, but resulted in some ALHAT navigation estimate issues that could not be quickly resolved. As a result, the LOS range measurements were not processed by the ALHAT navigation filter during the last three free flights, as shown in Figure 8.

Because of concerns that the heat from the VTB engine and the debris blown up during launch could damage the NDL and laser altimeter optics, debris shields were installed beneath the lenses. Acrylic debris shields were employed during FF10, but they deformed from heat during flight and resulted in poor NDL and laser altimeter availability. Polycarbonate material was used for the FF11 debris shields because of its much higher melting point, but the results were similar to those of FF10. The polycarbonate debris shields sagged from the heat, forming concave areas in front of the optics, and also tended to collect moisture and dust which further degraded the lidar signals. For FF 12 and beyond, the debris shields were eliminated in favor of cylindrical aluminum baffles to reduce the exposure of the lenses to debris strikes. The baffles worked well and had no adverse impact on sensor performance.

The attitude and pointing information from the ALHAT navigation filter and the VTB navigation filter were consistently close throughout the entire series of free flights, and the HDS was able to generate a high quality DEM of the hazard field during every flight. Based upon truth data, five safe landing areas exist within the 60x60m DEM generated by the HDS during the approach trajectory. However, because the Morpheus team wanted to land on a concrete pad, the hazard field was slightly modified for the Morpheus/ALHAT flights to increase the apparent safety of the landing pad relative to the other safe areas. During all five free flights the safe landing sites identified by the HDS were valid (always fell within the five areas known to be safe from truth data), and the HDS site ranked highest in terms of safety probability was always on the landing pad. The HDS safe site was used for FF12 and FF13, but on FF14 the safe site location was $0.5 \mathrm{~m}$ outside of the $2 \mathrm{~m}$ radius threshold imposed by the VTB to ensure touchdown on the concrete landing pad and was rejected by AFM.

The Morpheus GNC used the Morpheus navigation solution during FF10 through FF12. The ALHAT navigation filter executed "open loop" during these flights in order to gather performance data. Issues were identified and fixed after each of the three flights and the decision was made to close the loop on ALHAT nav for FF13 and FF14. The dual-string Morpheus/ALHAT navigation filter approach used by the Morpheus vehicle provides a unique and highly useful flight safety feature. The proven VTB nav always runs as a hot backup to the parallel nav string and the AFM controls which navigation output is routed to GNC to fly the vehicle. At any point in the trajectory that the ALHAT navigation estimate drifts outside of the boundary of the acceptable corridor defined around the VTB navigation estimate, the AFM switches back to VTB nav to complete the flight. This technique enables a new navigation approach to be safely tested in closed loop flight with far fewer flights than would normally be required since the proven VTB navigation filter is always ready to take over. On FF14, the ALHAT nav stayed within the defined VTB corridor throughout most of the flight, with AFM switching over to VTB nav just four seconds before the end of the effective corridor. Analysis after the flight showed that ALHAT nav would have safely landed the vehicle on the pad if it had been allowed to complete the flight.

HRN was the only ALHAT technique that was not fully verified during the Morpheus/ALHAT free flights. Only one or two HRN measurements (out of 8 to 10 opportunities) were collected and processed by the ALHAT navigation filter during each of the closed loop flights. Some HRN images were obscured by pixel pretriggering due to dust and could not be correlated with the DEM basemap. Other images did not meet the correlation threshold imposed by ALHAT to define a valid HRN measurement, or exceeded the correlation radius and produced no measurement. In addition, the processing of HRN measurements tended to drive the ALHAT navigation solution away from the VTB navigation solution. The reasons for this behavior are unclear, and the flight data is being carefully examined using flight data replay and GNC simulations. HRN is still considered to be a highly valuable technique for limiting the growth in surface-relative position errors during the time between safe site selection and terminal descent to a planetary surface. Understanding and fixing these HRN issues is a major priority for the team before the Morpheus/ALHAT system is flown again.

The five free flights of the ALHAT system on Morpheus occurred during a relatively short time span (just under two months) considering the complexity and scope of the endeavor. The number and duration of the test campaigns were limited, for the most part, by the stringent project funding profile. The time between free flights ranged from six 
days to two weeks, which provided very little time for indepth analysis of data, identification of beneficial or required changes, and the implementation and verification of updates to the Morpheus and ALHAT hardware, software, and procedures. Thanks to the dedication of a very good ALHAT/Morpheus team, FF13 and FF14 came very close to achieving full success with only a few issues left to understand and fix. The Morpheus/ALHAT team is continuing to focus on post-flight analysis and rework to prepare the system for follow-on flight campaigns in FY2015 should funding become available.

\section{ALHAT SYSTEM PERFORMANCE}

Completion of the current free-flight campaign has provided the ALHAT and Morpheus teams with a wealth of data. Overall, the integrated ALHAT/Morpheus systems performed very well and achieved a majority of the desired flight-test objectives. Detailed analysis of individual sensor and ALHAT Navigation (aka., ALHAT Nav) performance is still underway, and some revisions are in work for future playback analysis and potential future free flights. This section will provide an overview of the current state of the performance analysis for each of the ALHAT sensors and the ALHAT Nav filter. Data will be shown primarily from FF14, which was the second fully closed-loop flight of ALHAT, but discussions will include some details from FF10-FF13. The first closed-loop flight was FF13, and FF10-FF12 were open loop for ALHAT sensors.

The nominal flight trajectory used for all flights of ALHAT onboard Morpheus is shown in Figure 11. The flight profile went to a maximum altitude near 250 meters and traversed just over 400 meters to the landing site. The first half of the descent trajectory was designed to mimic a lunar-like approach trajectory with a 30 degree slant angle, as desired for ALHAT imaging with the HDS. The latter half of the descent implemented a divert to the selected safe landing site (pre-determined or ALHAT-determined based on the flight objectives).

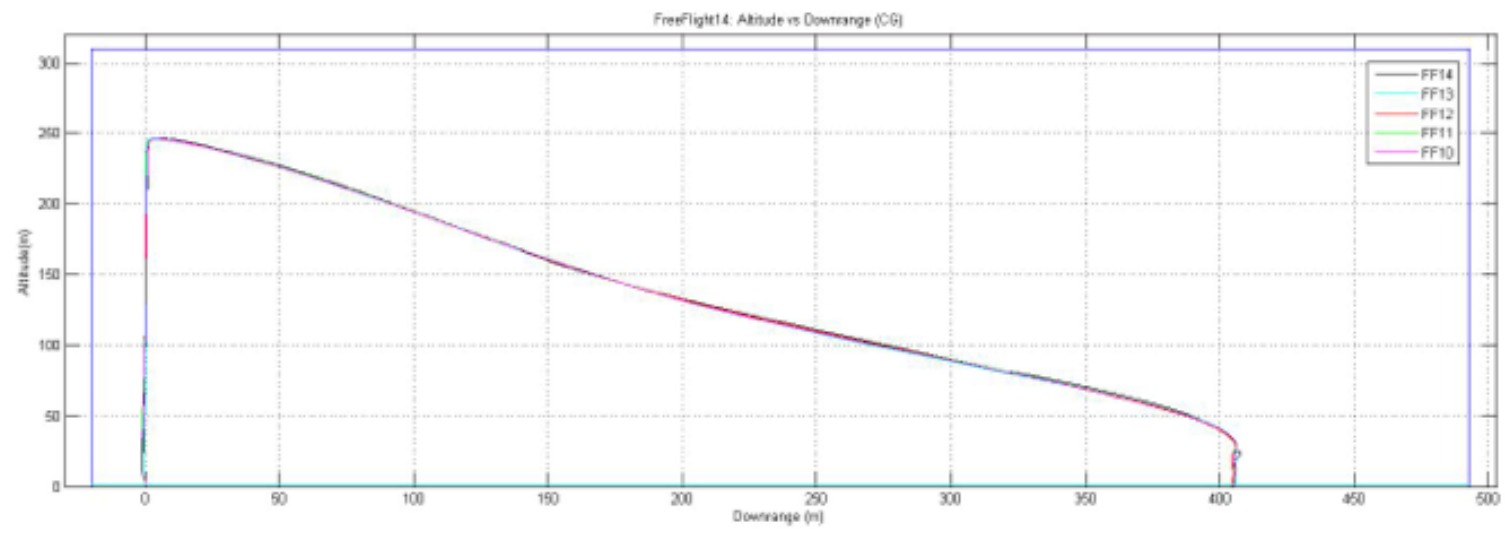

Figure 11. Flight trajectory profile for all ALHAT free flights onboard Morpheus

The timeline for the trajectory profile is visible within the FF14 LAlt data in Figure 12. As seen in the plot, the ascent portion of the trajectory is from approximately 15 seconds of Mission Elapsed Time (MET) after ignition until 40 seconds MET. The slant descent is from 40 seconds MET until $\sim 87$ seconds MET, and then the terminal

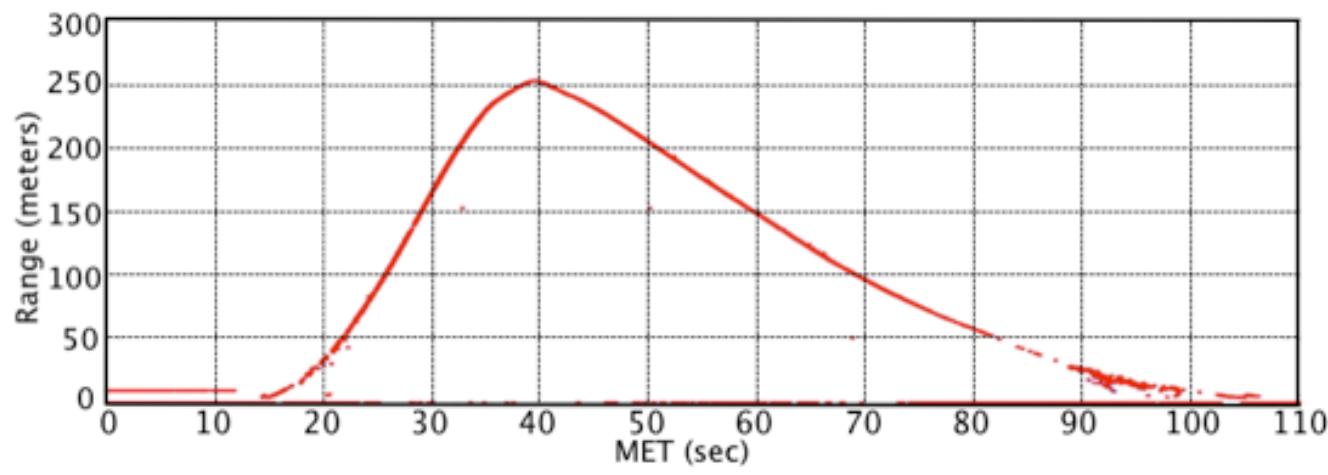

Figure 12. ALHAT Laser Altimeter data from Free Flight 14

American Institute of Aeronautics and Astronautics 
vertical descent from $\sim 35$ meters to the ground occurs after $\sim 87$ seconds MET. Overall, the LAlt produced consistent data throughout all of the free flights. Minimal measurement drops occurred during the flights, and the drops that did occur were mostly due to known degradation in the LAlt laser power that affected the signal to noise ratio; the laser will be replaced for any future flights. The 10 meter measurements prior to $\sim 12$ seconds MET occur when the sensor is at or below a 10 meter minimum range threshold; the LAlt is intended for use during TRN from very long ranges up to $20+$ kilometers at the Moon. The increase in measurement drops and noise after 85 seconds is believed to be primarily the result of dust kicked up during engine plume interactions with the ground.

The HDS provides three primary functions during the descent trajectory: 1) generate a Digital Elevation Map/Model (DEM); 2) conduct Hazard Detection (HD) on the DEM to determine and report safe landing sites to the vehicle Autonomous Flight Manager (AFM); and, 3) provide Hazard Relative Navigation (HRN) measurements to ALHAT Nav based on tracking a feature identified within the DEM. The DEM generation and HD performance was superb for all five free-flights of HDS on Morphues. Safe sites determined within the DEM were always safe in truth, and the top safe site was always on the truly safest region of the terrain. To find safe sites, the HDS FL measurements and HD algorithms were required to differential true roughness hazards of 30 centimenters or larger from FL range noise on the order of $8-\mathrm{cm}(1 \mathrm{~s})$. The hazard field was constructed so that the safest region would be on a concrete landing pad, which aids in landing site reusability and vehicle accessibility. The image and digital terrain in Figure 13 are of the hazard field, the outline of the DEM scanning region, and the actual 3-D construction of the DEM from HDS FL data obtained in FF11. The DEM from FF11 was the best of all five flights, due in part to a head wind that minimized dust and heated air from increasing noise in the FL range images.
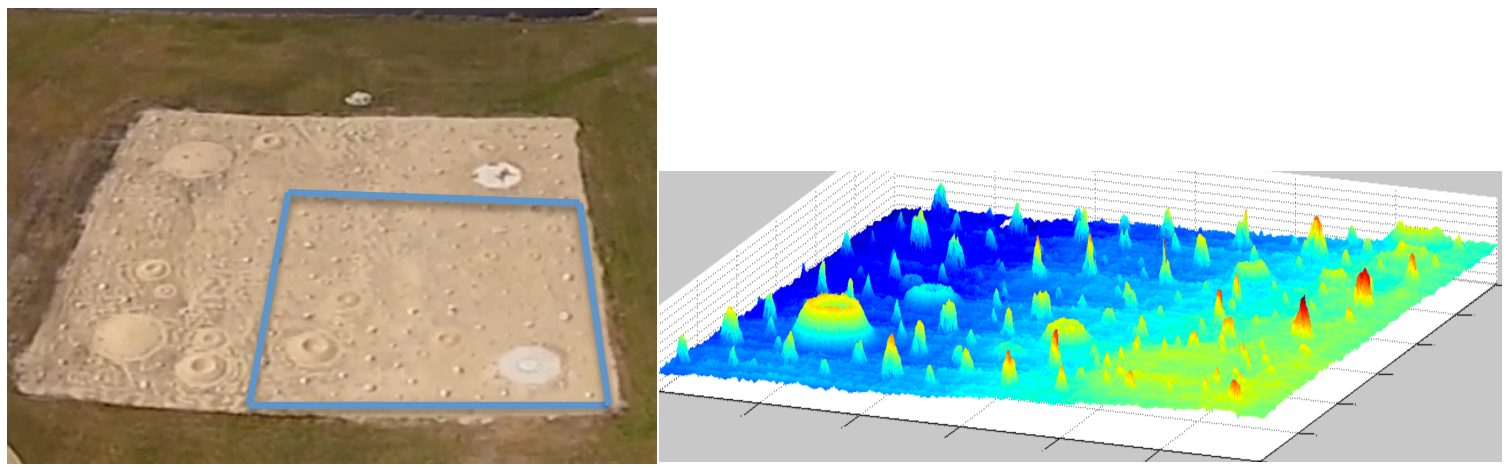

Figure 13. Hazard field with outlined scanning region (left) and Digital Elevation Map generated by the HDS during Free Flight 11

The FF11 DEM in Figure 13 is stretched herein to highlight the rocks and craters within the terrain map. The HD algorithm that runs in real time onboard the HDS processes this DEM to determine candidate landing sites that are ranked by safety probabilities. The HD algorithm makes use of geometric parameters for the lander, including vehicle and footpad dimensions, engine nozzle clearance, vehicle landing orientations, and slope tolerances for landing. Additionally, FL range precision and ALHAT Nav knowledge uncertainty are considered within the safety probability calculations used to locate and rank the safe sites. The top five ranks safe sites, as shown in Figure $\mathbf{1 4}$ for FF11, are sent into the Morpheus AFM for determination of which site will be used for the divert (always the top safe site for these flight test campaigns). The AFM also compares the HDS sites to known surveys of the top landing site to ensure the desired landing location is selected for the terrestrial test campaign. 


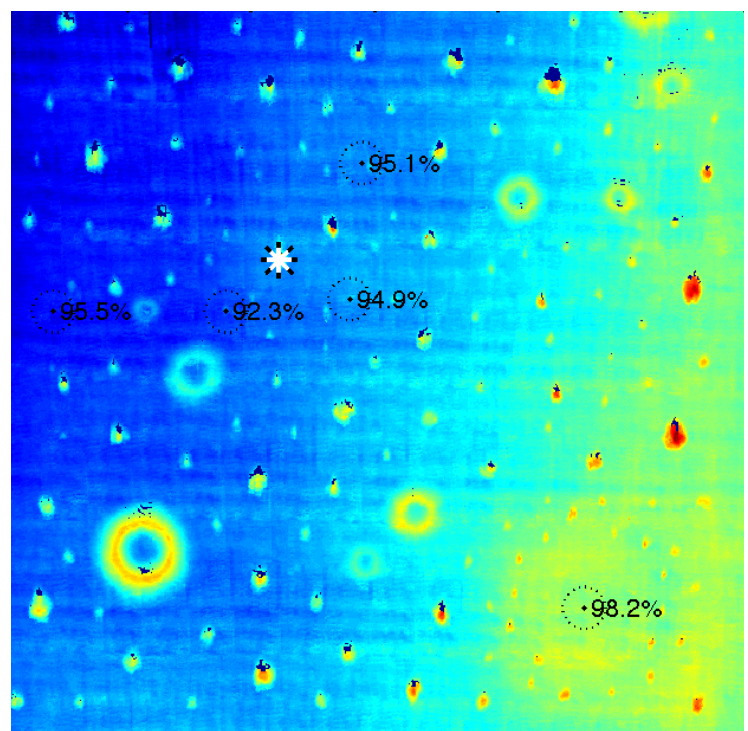

Figure 14. Free Flight 11 DEM with top five ranked safe sites (black dotted circles with \% safety probability) and selected feature for HRN tracking (white asterix within black dotted circle)

The HDS points the FL to the terrain field based on the pose estimate provided by ALHAT Nav for the HDS location relative to an Intended Landing Point (ILP). The ILP is an a priori landing location typically chosen from coarse orbital reconnaissance data early in a mission. The DEM is centered on the ILP, and coordinates of the locally determined safe sites are provide in an ILP coordinate frame. Knowledge error in the ALHAT Nav estimate of ILP-relative HDS pose causes a map tie error between the DEM and the pre-mission desired mapping region. This is a known phenomenon and is not a problem in the architecture of ALHAT Nav, and HRN measurements are used after HD to minimize the additional growth of DEM-relative errors. This map tie does however caused issues with terrestrially testing ALHAT since the Morpheus AFM, which also is unaware of the map tie shift, compares the HDS top safe site with a truth survey. To accommodate the error, an artificial 2-meter VTB threshold is allowed for the ALHAT determined safe site, as shown in Figure 15. Again, this is a terrestrial test constraint for Morpheus flights and does not impact ALHAT Nav performance.

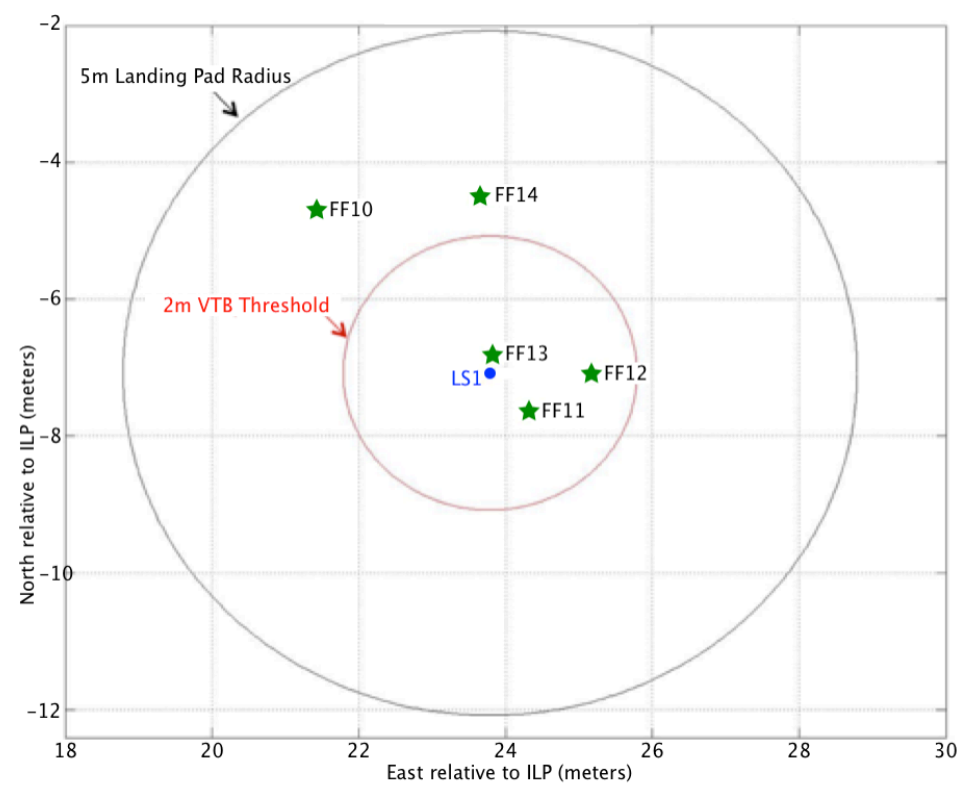

Figure 15. AFM comparison of HDS reported safe sites against the truth survey of the pad center (LS1). Safe sites for FF10 and FF14 where outside of the 2-meter VTB constraint. This terrestrial test constraint is impacted by map tie error that does not affect ALHAT Nav.

18

American Institute of Aeronautics and Astronautics 
For FF12-FF14, the Morpheus AFM selected either the HDS determined safe site or the surveyed safe site for the guidance divert for vehicle landing. The surveyed site was planned for use in FF10 and FF11. In both FF12 and FF13, the top HDS site was within the VTB threshold and was selected. In FF14, the site was outside of the threshold, so the surveyed landing site (LS1) was used instead. After the safe site selection, the HDS transitions into HRN feature tracking and provides HRN measurements that provide ALHAT Nav with DEM-relative position updates to minimize position error growth after safe site determination. More details on HRN will be covered later in this section.

The NDL has three independent beams that provide Line of Sight (LOS) velocity and range measurments. During the flight testing, the location of the NDL subjected the sensor beams to excessive heated air and thermal gradients. This caused drops in certain beams based on the wind direction for each flight, as visible in Figure 16 for FF14. In the plot, Beam 1, the forward beam, drops out during $\sim 25-45$ seconds MET, which corresponds to the ascent portion of the flight. Beams 2 and 3, which point 120 degrees aft in opposite directions, drop out over flight segments from the peak of the trajectory until midway through the descent. Similar to the LAlt, the loss of all three NDL beams after 90 seconds is believed be the result of the engine plume kicking up dust at the landing area.

During the open-loop flights, FF10-FF12, the NDL measurement drops and less than three simultaneous beams appeared to coincide with odd behavior and apparent sensitivities within the ALHAT Navigation filter. During the closed-loop flight testing in FF13 and FF14, the NDL range measurements were excluded and additional requirements and constraints were put in place to govern when NDL velocity measurements were processed. For FF13 the NDL velocity data was only processed after the HDS generated its Digital Elevation Map (DEM) to ensure a well behaved Navigation state for HDS pointing and slewing. For FF14, the NDL measurement processing was further constrained to require that all three beams had velocity measurments. In depth analyses including playback of flight data are currently underway to fully understand the filter interactions with the NDL and other ALHAT measurements to resolve the processing issues.

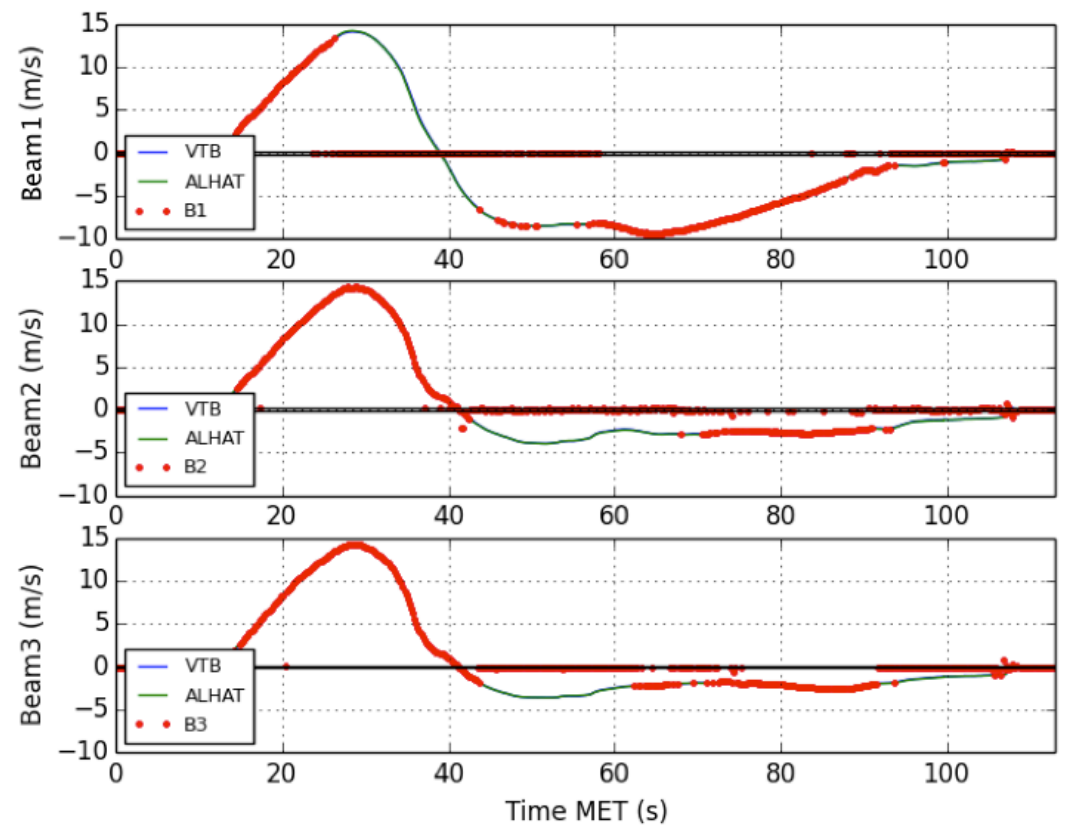

Figure 16. Navigation Doppler Lidar data from Free Flight 14

The ALHAT Nav filter processes measurements from the HDS, LAlt and NDL, along with IMU data from the Morpheus SIGI. The filter provides the glue between the ALHAT system and the Morpheus GN\&C functions. The Morpheus GN\&C implements a dual-string Nav architecture that runs both the ALHAT Nav filter and a VTB Nav filter (based on GPS, SIGI and other non-ALHAT sensor data). The VTB Nav runs in the background and is used by the Morpheus AFM to monitor the ALHAT Nav state. This dual-string architecture enables the Morpheus VTB to safely test and rapidly mature ALHAT (or other prototype GN\&C) technologies. In the event that ALHAT Nav 
violates state-threshold criteria relative to VTB Nav, the Morpheus AFM switches the guidance and control loop from ALHAT Nav to VTB Nav to ensure vehicle safety; this is called downmoding. There are threshold corridors for position, velocity and attitude: Figure 17 shows the position threshold corridor, along with the ALHAT versus VTB position difference for FF14.

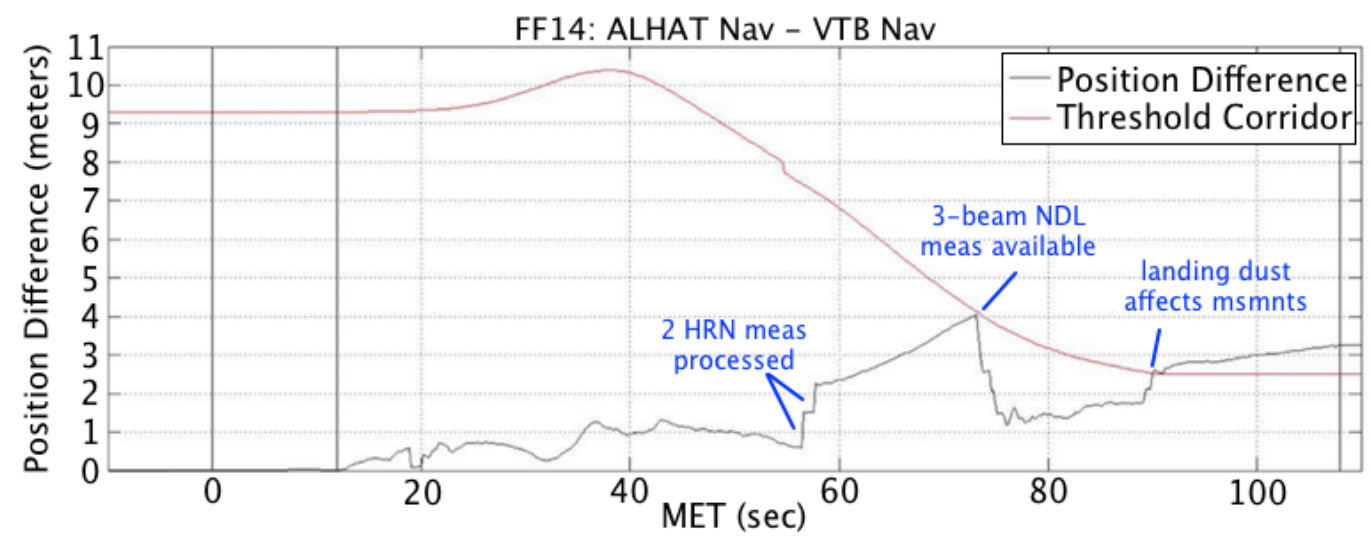

Figure 17. Position threshold corridor used to monitor ALHAT Nav versus VTB Nav. Data shown for FF14.

The data in Figure 17 shows the position difference between ALHAT Nav and VTB Nav, along with the position threshold used by AFM for downmoding. Several events are noted in the figure, including locations where HRN measurements were processed, as well as all NDL measurements being available (for FF14). In both closed-loop flights, FF13 and FF14, there was an AFM downmode event. The downmode in FF14 is near 90 seconds MET, and the downmode in FF13 was at 67 seconds MET. The FF14 downmode was within the final 25 meters of terminal vertical descent where engine plume interaction with the surface kicked up dust that adversely affected the NDL and LAlt measurements processed by the ALHAT Nav filter. To handle dust interactions, ALHAT Nav implements a cutoff altitude below which the IMU is used for dead reckoning and NDL and LAlt measurements are not processed. The FF14 cutoff was set based on observations in prior flights which turned out to not be conservative enough. This is not deemed an issue for assessing overall ALHAT performance since nominal lunar descent plans a 30 meter cutoff altitude (or higher based on continued analysis).

The difference data in Figure 17 shows that only two HRN measurements were processed, followed by a trend toward the threshold. When 3-beam NDL data comes in near 75 seconds MET, the difference data attains significant margin from the threshold. This growth trend after HRN processing is a source of ongoing analysis. The team is currently investigating both the production of HRN measurements within the HDS and the processing of those measurements within ALHAT Nav. Additionally, basic tuning analysis of the filter is underway to determine if measurement weightings are causing odd filter behavior. There is a current requirement in the measurement processing to only accept NDL measurements when all three beams are available. This requirement was due to a similar walkoff in FF13 that caused a downmode to VTB Nav at 67 seconds MET. The quick turnaround analysis between FF13 and FF14 identified the filter sensitivities to dropouts in NDL data, which drove the prior mentioned constraint of requiring all NDL beams for filter processing. The walkoff in FF14 indicates other filter or measurement processing sensitivities, so analysis is ongoing to fully understand the behavior in both simulation and playback of flight data.

The HRN capability is a key innovation within ALHAT to enable precision landing to within 1-meter (1s) of the determined safe landing site. The HRN algorithms within HDS and the measurement processing by ALHAT Nav was an untested feature prior to the Morpheus flight tests, so the teams conducted cutting-edge technology development during the campaign. Significant progress toward maturing HRN was accomplished during the campaign, and continued analysis is underway. The HDS was able to accurately point to the desired terrain feature during initial HRN measurments, but closed-loop pointing between HDS and ALHAT Nav prevented successful HRN after the first two measurements on both FF13 and FF14. Some insight has been provided by the ALHAT Nav response to HRN measurements in Figure 18. These errors may not be related to a filter formulation error, but rather filter sensitivies to tuning and weighting of other ALHAT sensor measurements or errors within the measurements themselves since they are derived from HDS comparisons between the FL images and the DEM. The team is actively analyzing this phenomenon to refine HRN and overall filter tuning for future flight testing. 


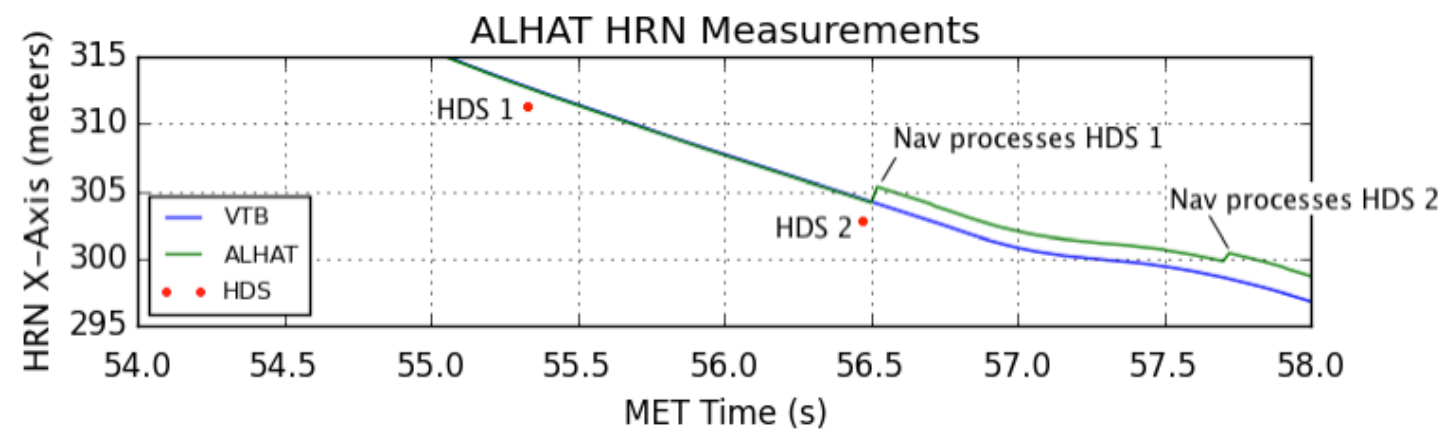

Figure 18. Current HRN processing indicates filter sensitivities to HRN measurements or potential errors within the measurements themselves. Analysis is underway to determine the root cause.

\section{ALHAT COMPONENT AND SYSTEM MATURITY}

Two ASC Tiger Eye flash lidar hazard detection sensors were used by ALHAT for the all of the field tests since the beginning of the project. These were purchased in 2007 with one used for early field testing and all of the helicopter flight tests. Over the course of this testing this flash lidar had over a thousand pixels go bad due to mosture condensation on the array. By masking these bad pixels in software the imaging software could still give good images but as the number increased the quality of the images also decreased. Because of this, the second flash lidar with less than fifty bad pixels was calibrated and utilized in the Morpheus flight testing. To avoid the moisture condensation issue, a purge of dry nitrogen was used whenever the dew point temperature was within 2 degrees of the ambient temperature. This worked well except during final vehicle flight preparation and flight when it was not possible to keep the purge on the lidar. Thus, there was some pixel damage during the night flight and night flight dry run due to the dew point temperature but was still limited in scope and the lidar with pixel masking continued to function well. There were some bad pixels around the edges of the array during flight but not during static conditions, the cause of which is not fully explained. It was assumed to be due to the vibration environment during flight so these pixels were always masked after the effect was identified on the first flight. With this masking and very good calibration this flash lidar gave very good images of the hazard field with very good DEMs.

Flash lidar technology has improved considerably since the ALHAT Project purchased them and some have flown in space. The ALHAT/Morpheus flights demonstrated the viability of flash lidars as hazard detection sensors for real-time hazard detection and avoidance for planetary landings. Thus the utilization of flash lidar sensors for planetary landing HDA is considered to be at TRL 6 or higher with the understanding that any flight lander would need to certify their unit for spaceflight.

The Navigational Doppler Lidar (NDL) is not commerically available and was developed at the LaRC. During the ALHAT/Morpheus flights the hot air plume from the rocket engine caused considerable drop outs of the NDL beams during flight. This effect was not known preflight and the location of the NDL on the under side of the vehicle was problematic. In future terrestrial flights this should be a major consideration. Even with these environmental issues the NDL provided excellent velocity data during portions of the flights. Based on these Morpheus flight tests and the other ALHAT field testing of the NDL it is considered to be at TRL 6. It will require some refinements in components to reach space certification and it is well known that the size, mass, and power could and should be reduced considerably before it is viable for a space mission. It should be noted that the NDL can also measure altitude and attitude but was not utilized for that purpose during the ALHAT testing.

The ALHAT laser altimeter was designed and built at LaRC. It has been tested to $30 \mathrm{~km}$ in the earth atmosphere and by extroplation is expected to work in space to at least $50 \mathrm{~km}$ with accuracies on the order of $30 \mathrm{~cm}$ with some dependence on distance. This sensor also is not commercially available but has proven to be an excellent landing sensor. It is considered to be at TRL 6 or higher but again would require some refinements in components to reach space certification and the size, mass, and power should be reduced before it is ready for spaceflight. 
The TRL 6 for the three sensors above includes both the hardware design and function as well as the associated software. All of the software proved to be robust and is ready to be taken the next step for spaceflight certification.

The Hazzard Detection System (HDS), designed and developed at JPL, uses the data from the flash lidar to build a DEM of the hazard field and identify safe sites. This system running on a special multicore processor worked very well on helicopter and the ALHAT/Morpheus flight tests in identifying safe landing sites. This system is also used to perform HRN and while we were able to get a few HRN data points it did not work as expected. It is unclear at this time where the problem lies but several items are under investigation and expected to be fixed for any future flight tests. The hazard detection and safe site selection is definitely at TRL 6 and the HRN technique should be at TRL 6 once the problem is fixed.

The ALHAT navigation Kalman developed at JSC was finalized shortly before it was put into service for the ALHAT/Morpheus test flight campaign. Overall the filter worked very well except in the HRN function and the utilization of the NDL velocity data. Again, it is unclear at this time where the problems with these two items reside but considerable effort is going into identifying the problems and getting them fixed before the next flight demonstration. It could be a matter of tuning the filter or getting the timing correct in the measurement data. This is a very stable filter and works well and should be at TRL 6 once these issues are understood.

The ALHAT techniques of TRN, HDA, and HRN have all been demonstrated and are at TRL6 or higher. Also, the technique of using precision terrain relative sensors to maintain very precise navigation states during approach and landing which allowed dead reckoning to the surface during the last $50 \mathrm{~m}$ of descent, have been demonstrated and are considered to be at TRL 6 or higher. Thus, all of the ALHAT stystems and techniques are considered to be at the TRL 6 level and ready to be utilized in planetary missiona pending the resolution of the two issues described above.

\section{THE FUTURE OF ALHAT}

The ALHAT Project has brought autonomous precision landing and real-time hazard detection and avoidance with safe landing to the planetary landing technology forefront. The decision from inception was to develop a completely general system that would work for any lander under any lighting conditions. Because of the any lighting conditions constraint, all of our systems use active laser sensing systems. However, it was clearly understood that removing the any lighting conditions constraint opened up the utilization of passive camera systems to perform some of the ALHAT functions. In particular, TRN was demonstrated on FT3 with two different passive camera systems and both worked well with appropriate lighting. There are also some HDA techniques using passive cameras that have been suggested but they generally are limited in capability. In addition, in the past few years some very high speed scanning lidars and hybrid lidars have been developed that could be considered for the HDA and HRN functions.

The long term goal of the ALHAT Project is to encourage and support the utilization of ALHAT techniques on planetary landing missions. These technologies have a lot to offer and should be considered in planning any future landing mission. It appears that many of the future planned robotic landers will use some form of TRN for precision landing. This is a relatively easy addition to robotic missions and will provide the capability of landing close to the premission defined location. Most of them will be performed using passive optical cameras.

There is also considerable interest in adding Doppler lidar velocimetry to some of the robotic missions that are in planning. The ability to precisely measure the lander ground relative velocity of a landing vehicle is a very valuable navigation asset. The ALHAT project is seeking funding to construct a flight certifiable Doppler lidar unit that can be available for future robotic missions. The ALHAT project is also seeking funding to construct a flight certifiable laser altimeter which also is a very valuable navigation asset during approach and landing, particularly at higher altitudes.

The next step in the evolution of ALHAT technologies is the addition of real-time hazard detection and avoidance during the last 1000 meters from landing. This technology has more cost and risk associated with adding it to a landing mission but offers high probability for a safe landing particularly in areas that are somewhat hazardous and possibly of great scientific interest.

The NASA investment of over $\$ 80 \mathrm{M}$ in full cost dollars over approximately nine years has opened the planetary lander technology to any planned mission and in the future will prove to be a good investiment as these technologies 
get utilizied in landing missions. All of these technologies are tailorable to specific missions including techniques and sensors.

\section{Acknowledgments}

The authors gratefully acknowledge the tremendously awesome ALHAT/Morpheus team including members from JSC, JPL, KSC, LaRC, IM, Helios, CSDL, SSC, and Marquette University, who supported the ALHAT system flight demonstrations on the Morpheus vehicle in FY2014. This cross NASA center and contractor team showed unsurpassed dedication and support for all systems and activities working together as one to make the demonstrations successful. There are stories of amazing sacrifice and going beyond expectations by many members of the team and all are greatly acknowledged.

\section{References}

Proceedings:

${ }^{1}$ Epp, C.D. and Smith, T.B., “Autonomous Landing and Hazard Avoidance Technology (ALHAT)," in Aerospace Conference, 2007 IEEE, 2007, pp. 1-7.

${ }^{2}$ Epp, C.D., Robertson, E.A., and T. Brady, “Autonomous Landing and Hazard Avoidance Technology (ALHAT)," in Aerospace Conference, 2008 IEEE, 2008, pp. 1-7.

${ }^{3}$ Rutishauser, D.K., Epp, C. D.,Robertson, E.A., "Free-Flight Terrestrial Rocket Lander Demonstration for NASA's Autonomous Landing and Hazard Avoidance Technology (ALHAT) System”, AIAA SPACE 2012 Conference \& Exposition. September

${ }^{4}$ J.M. Carson, E.S. Bailey, N. Trawny, A.E. Johnson, V.E. Roback, F. Amzajerdian, and R.A. Werner. "Operations concept, hardware implementation and ground-test verification of a hazard detection system for autonomous and safe precision lunar landing". AAS/AIAA Astrodynamics Specialist Conference, Hilton Head Island, SC, August 11-15, 2013.

${ }^{5}$ Diego F. Pierrottet, Farzin Amzajerdian, Larry Petway, Glenn Hines, and Bruce Barnes. "Field Demonstration of a Precision Navigation Lidar System for Space Vehicles". AIAA Guidance, Navigation, and Control Conference, Boston, Massachusetts, August 19-22, 2013.

${ }^{6}$ Farzin Amzajerdian, Diego Pierrottet, Larry B. Petway, Glenn D. Hines, Vincent E. Roback, Robert A. Reisse. "Lidar Sensors for Autonomous Landing and Hazard Avoidance" AIAA Space2013, San Diego, CA, September 10-12, 2013.

${ }^{7}$ John M. Carson, Nikolas Trawny, Andres Huertas, Michael Luna, Vincent E. Roback, Andrew E. Johnson, Keith Martin. "Performance of a Real-Time Hazard Detection System for Lunar Landing". AIAA Space2013, San Diego, CA, September 1012, 2013. 